\title{
CATALOGUE OF FRUITS
}

ror

CULTIVATION IN THE UNITED STATES AND CANADAS;

\section{IN TWO DIVISIONS.}

DIVISION FIRST-Embracing those States lying North of the Southern line of V'rrginia, Tendessee, Missouri, etc., and East - of the Rocky Mountains, including the Canadas.

DIVISION SECOND-The States Socth of the Line above Named, and West of the Rocky Mountains.

COMPILED UNDER THE DIRECTION OF THE

\section{AMERICAN PONOLOGICAL SOCIETY,} FIOM THE

disparts of State and Bistrict Cummittes.

INTENDED TO KXHIBIT, IN A FORM CONYENIENT FOR REFEREXCE, TIE VARIETIES OF FRUITS BEST ADAPTED TO TIIE DIFFERENT STATES AND LOCALITIES: SUBJECT TO FUTURE REVISION AND CORRECTION, AS EXPERIEỴCE MAY RENUER NECESSARY AND PROPER.

\section{REVISED E D I T ION.}

PUBIISHED PY IIE SOOIETY.

B OSTON:

SAM'L CHISM, - FRANKLIN PRINTING HOUSE, No. 134 WASHINGTON STtete

1868 . 



\section{CATALOGUE OF FRUITS}

FOR

CULTIVATION IN THE UNITED STATES AND CANADAS;

IN TIVO DIVISIONS.

DIVISION FIRST-EMbracing those States Lying North of the Southern line of Virginia, Tennegsee, Missovri, etc., and East of the Rocky Mountains, including the Canadas.

DIVISiON SECOND-The States South of the line above Named, and West of the Rocky Mountalns.

COMPILED UNDER THE DIRECTION OF THE

\section{AMERICAN POMOLOGICAL SOCIETY,}

FROM THE

disports of State and Distrist Committers.

INTENDED TO EXHIBIT IN A FORM CONVENIENT FOR REFERENCE, TILE VARLETIES OF FRUTT BLST ADAPTED TO THE DIFFERENT STATES AND LOCALITIES:

SUBJECT TO FUTURE REVISION AND CORIECTION, AS EXPERIENCE MAY RENDER NECESSARY AND PRUPER.

REVISED EDITION.

BOS TO N :

SAM'L CHISM, -FRANKLIN PRINTING HOUSE, No. 134 WASHINGTON STREET.

1868 . 



\section{PLAN OF TIIE CATALOGUE.}

TuE arrangement of the varieties is alphabetical, according to the nomenclature adopted by the Society. Synonyms are given in a few instances only, where it seemed necessary; and these are placed under the arlopted name, in italics. The tabular arrangement has been adopted to obviate the necessity of repeating the names of varieties several times, as would be required if a separate Catalogue were made for several sections.

It has been decider by the Committee not to attempt, at this time, to give descriptions of the fruits recommended, but simply to indicate the season of maturity, use, and a few other particulars of importance, which require but little space. In regard to the season, it will be observed that, instead of naming the month or months in which fruits mature, the general terms "Summer," "Autumn," and "Winter," have been nsed; the peculiar arrangement of the Catalogue renders it impracticable to be more exact in this particular. For example: The Early IIarvest Apple is reported as succeeding in almost every State and Territory in the Union, in one locality ripening in May, and in another in July, or even in Angust, - a difference of two months or more; and yet in every locality it is strictly an eurly summer apple.

The columns are arranged thus: First, the names of varieties; next, the season, nse, etc.; and then the States or Districts, the names of which stand at the head of the columns.

The State or District in which any variety is recommended, is designated by a star (*); and in cases where a variety is recommended as being of great superiority or value in any locality, the indication is two stars, thus (**).

It being necessary to carry the column arrangement across two pages, numbers are used on the margin of each page, to prevent errors in following lines.

\section{DIVISION FIRST.}

Embracing those States north of the southern line of Virginia, Temnessee, Missouri, etc., and east of the Rocky Mountains, including the Canadas. 



\section{I. - A P PLES.}

Explanation of Colums. - The first column on the right of the name gives the season of maturity. The second, the particular nses for which the viriety is best adapted; and the remainder the Districts in which the varieties are recommended.

Explanation of Abrreviations. - Seasons: S. summer; A. autumn; W. winter; E. early; L. late. Those not designated as early or late of their season may be regarded as medium.

UsE. - K. designates varieties recommended only for kitchen purposes. C. those specially for cider. Those not marked $\mathbf{K}$. or $\mathrm{C}$. may be regarded as dessert or table sorts. MI. designates those most valued for market.

* Star opposite a variety indicates that such a variety succeeds well in the District named at the head of the column.

** Two stars distinguish those most highly reeommended. 


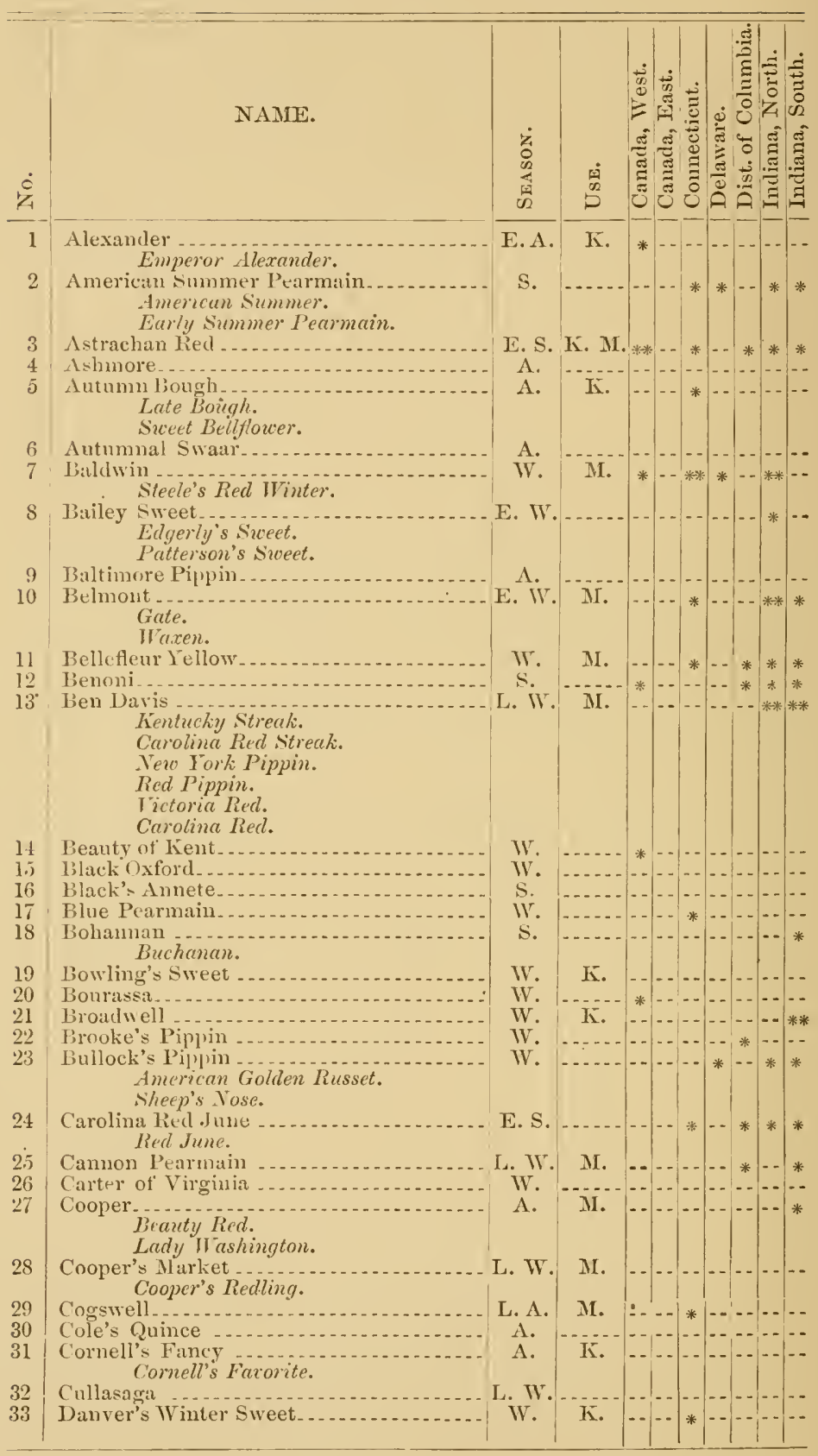




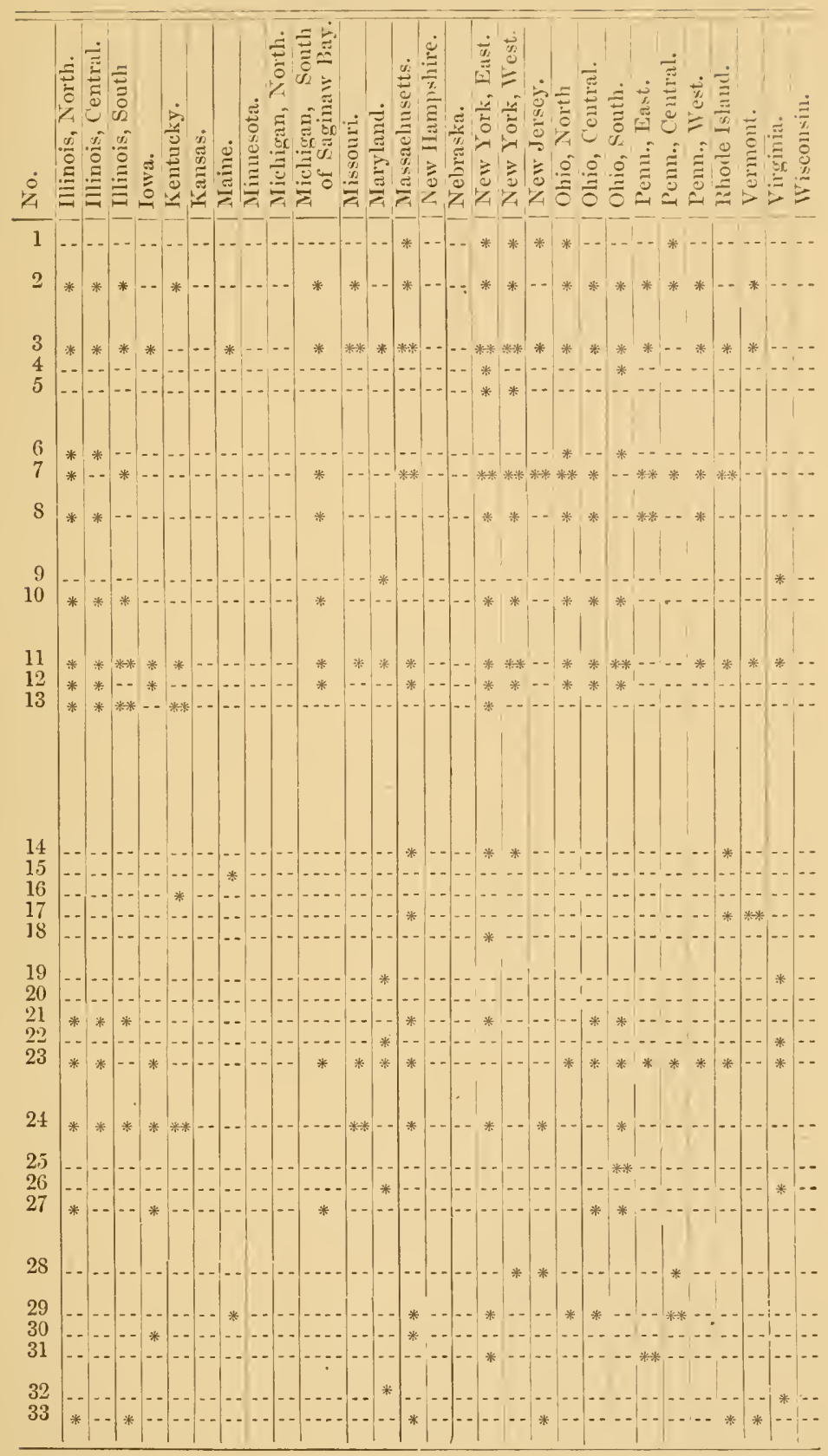




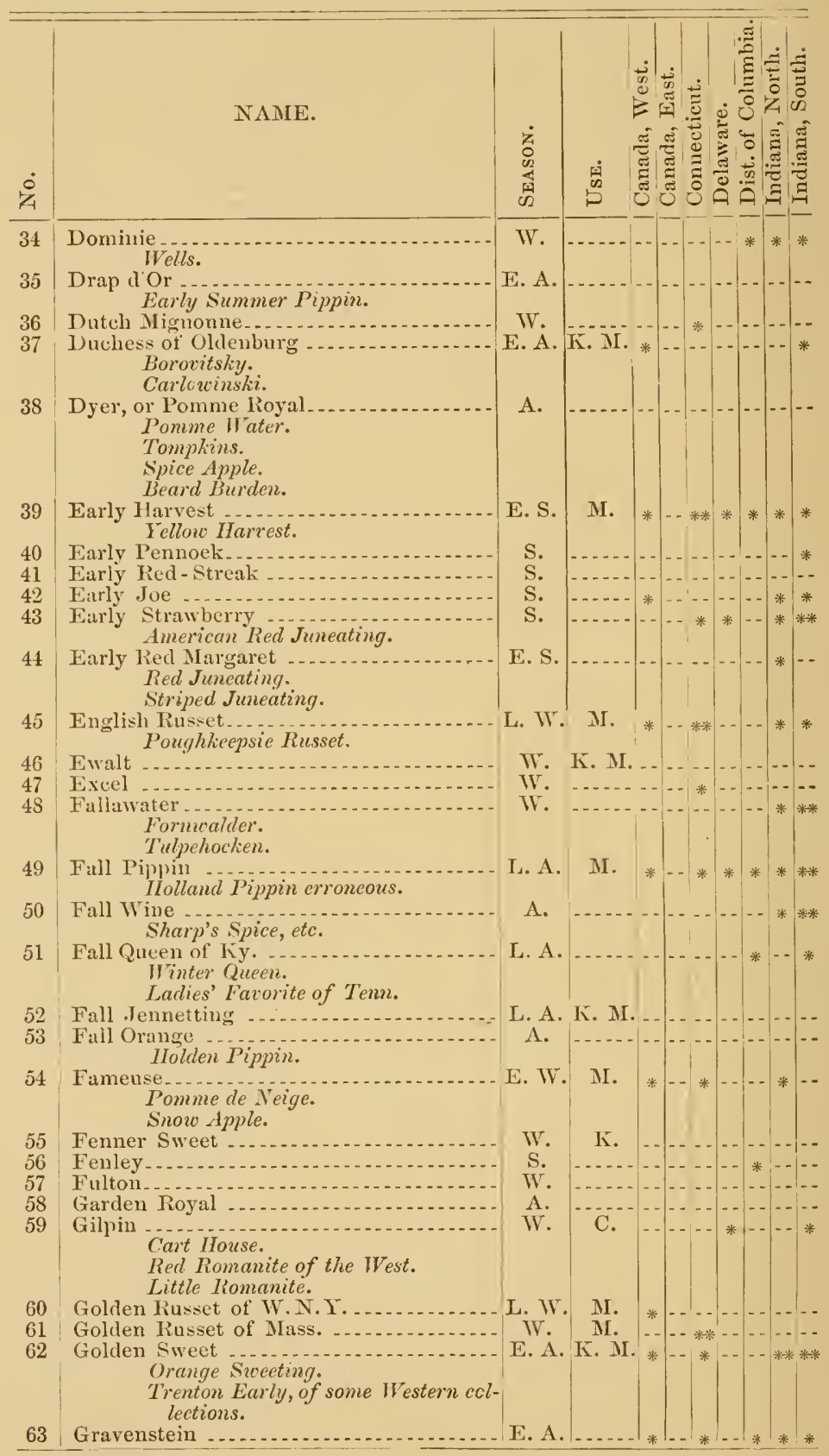




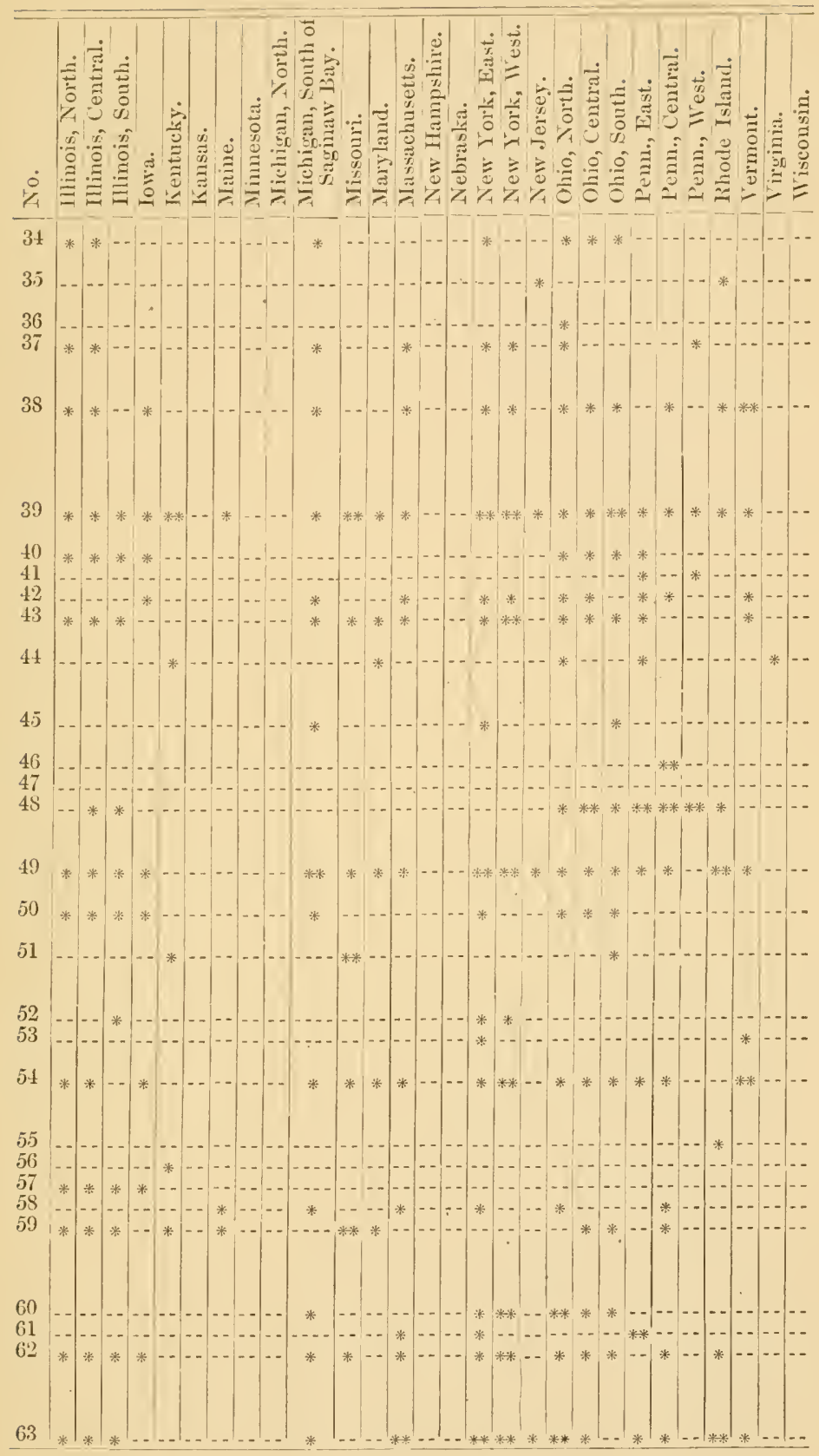




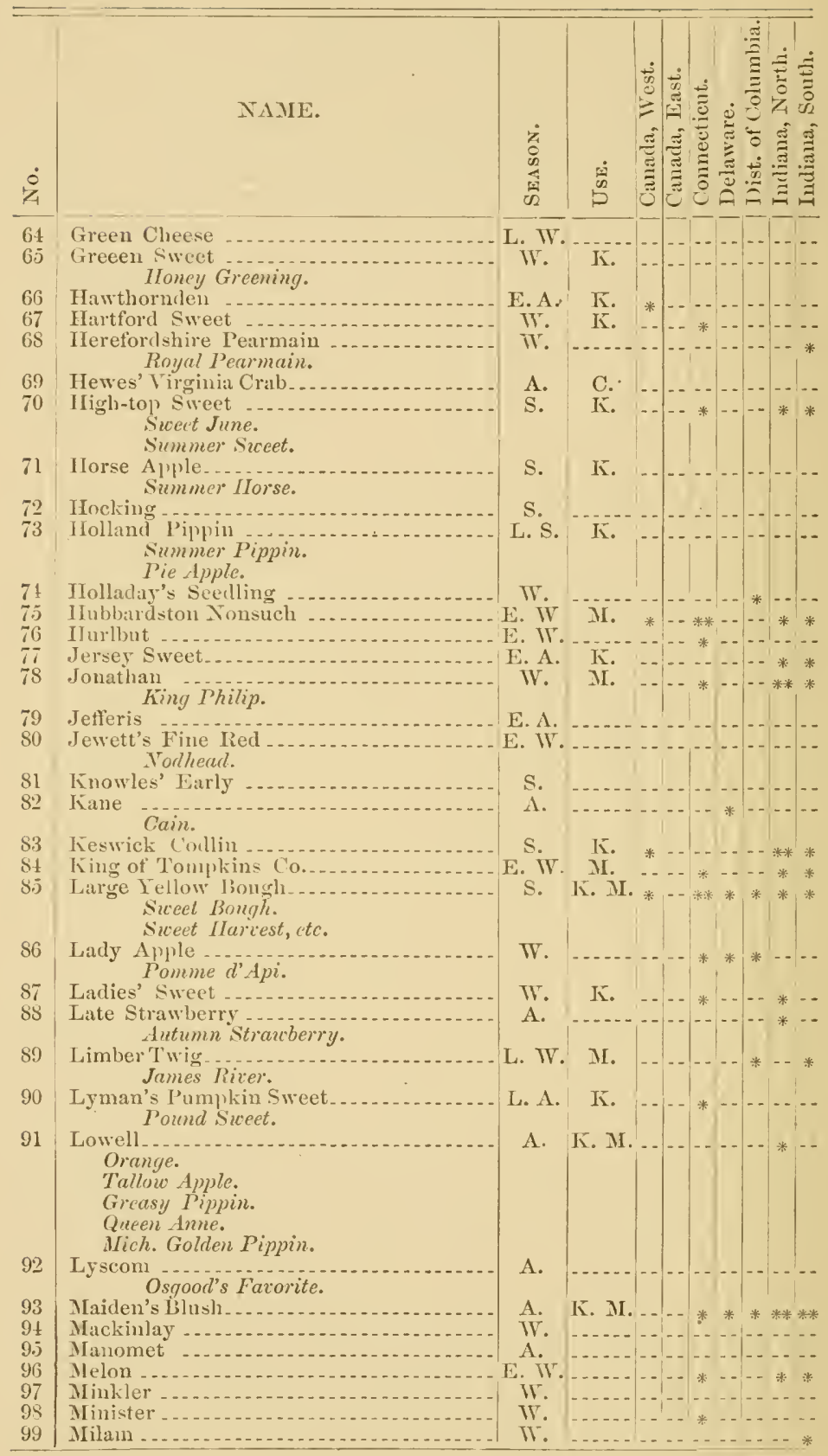




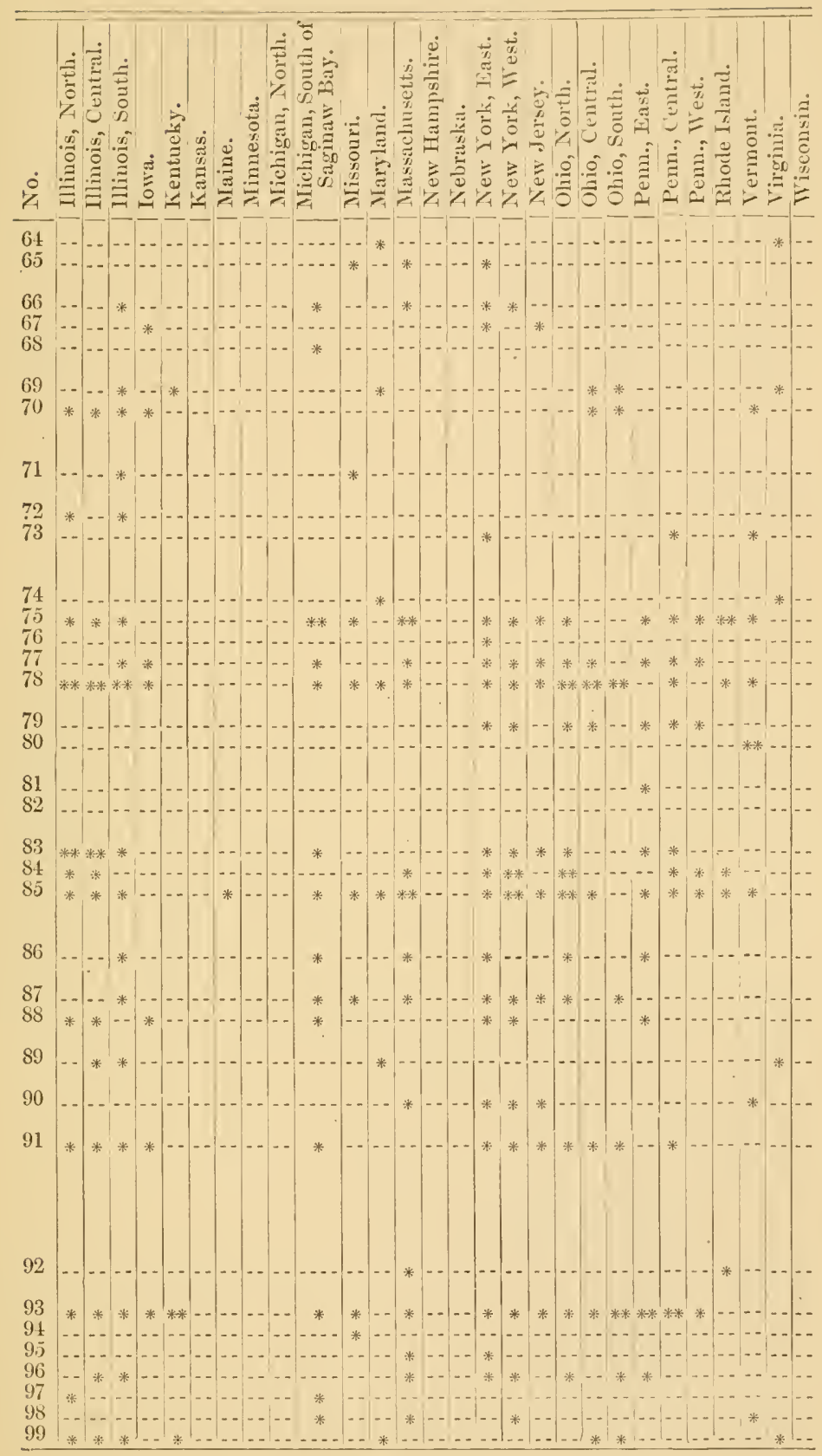




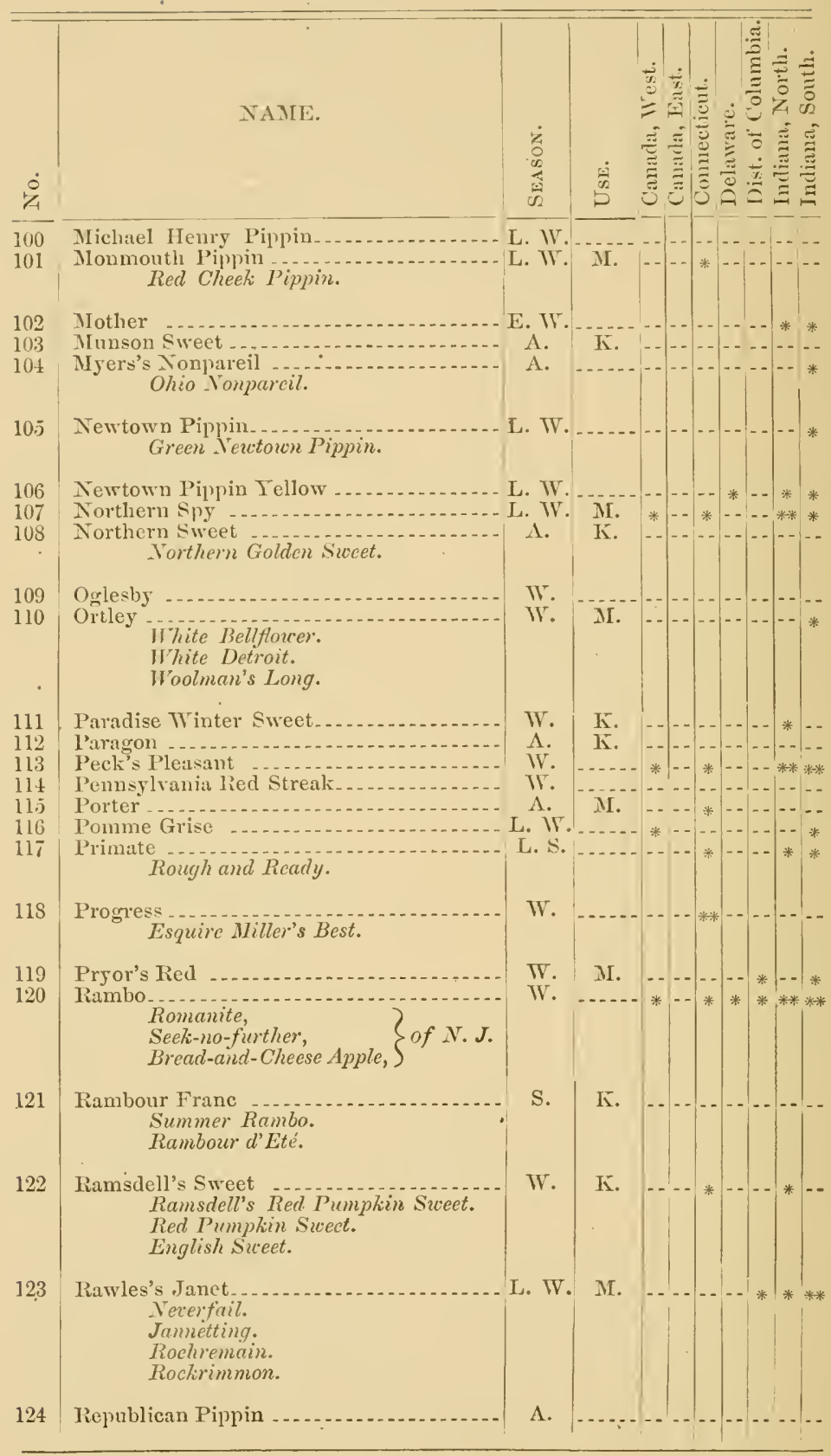




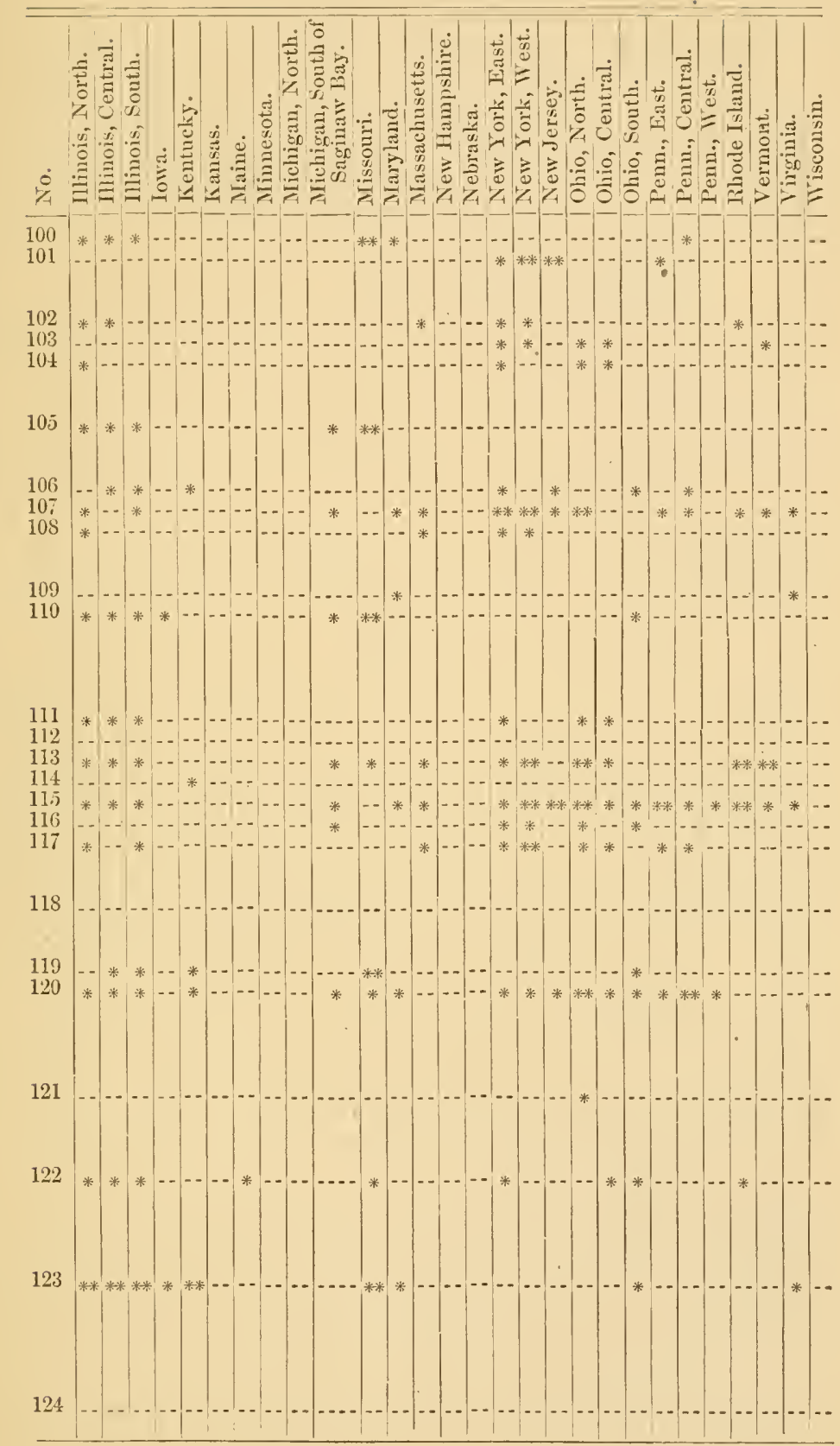




\begin{tabular}{|c|c|c|c|c|c|c|c|}
\hline 学 & NAJIE. & 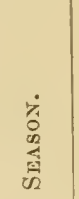 & $\frac{\dot{s}}{b}$ & & 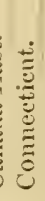 & & 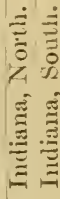 \\
\hline 125 & $\begin{array}{l}\text { Red Canada } \\
\text { Old Nonsuch of Mass. } \\
\text { Richfield Yonsuch. } \\
\text { Steele's Red Winter, of some collec- } \\
\text { tions in Mich. }\end{array}$ & W. & & * & & & \\
\hline $\begin{array}{l}126 \\
127\end{array}$ & $\begin{array}{l}\text { Red Ingestrie } \\
\text { lied Sweet }\end{array}$ & A. & K. & & & & \\
\hline & Red Winter $S$ & & & & & & \\
\hline 128 & Red Cathead. & W. & & $\ldots$ & & & \\
\hline 129 & $\begin{array}{l}\text { Reinette Canada } \\
\text { Canada Reinette. }\end{array}$ & WV. & & * & & & \\
\hline 130 & Rhode Island Greenin & W. & M. & * & * & & $*$ \\
\hline 132 & Ridge Pippin ... & L. W. & II. & - & - & & \\
\hline $\begin{array}{l}133 \\
131\end{array}$ & $\begin{array}{l}\text { Roman stem } \\
\text { Roxbury Russet }\end{array}$ & L. W. & II. & - & & & $*$ \\
\hline & $\begin{array}{l}\text { Poston Russett. } \\
\text { Putnam Russett, of some Western } \\
\text { collections. }\end{array}$ & & & & & & \\
\hline 135 & $\begin{array}{r}\text { Rome Beanty, } \\
\text { Gillett's Seedling. }\end{array}$ & W. & Mr. & $\mid-$. & & & $* * *$ \\
\hline 136 & Robey's Seedling- & W. & & . & & & \\
\hline $\begin{array}{l}137 \\
138\end{array}$ & $\begin{array}{l}\text { Robertson's W'hite } \\
\text { Saint Lawrence. }\end{array}$ & E. W. & & $*$ & & & * \\
\hline 139 & Sheppard's Sweet & A. & K. & & & & * \\
\hline $1+0$ & Sine qua non...... & S. & 1 & & & & \\
\hline $\begin{array}{l}1+1 \\
142\end{array}$ & $\begin{array}{l}\text { Smoke-house } \\
\text { Mill Creek Tandevere. } \\
\text { English Vandevere. }\end{array}$ & E. W. & $\begin{array}{l}\text { K. II. } \\
\text { jI. }\end{array}$ & & $*$ & & \\
\hline 143 & $\begin{array}{l}\text { Sops of Wine } \\
\text { Pie Apple. } \\
\text { Washington. }\end{array}$ & s. & K. & & & & \\
\hline $14 \pm$ & Spice Sweet & $\Lambda$. & K. & $\cdots$ & & & $* \ldots$ \\
\hline $\begin{array}{l}145 \\
1 \neq 6\end{array}$ & $\begin{array}{l}\text { Spitzenburg Esopus ... } \\
\text { Summer Queen }\end{array}$ & W. & I. & * & $*$ & & $* *$ \\
\hline 147 & $\begin{array}{l}\text { Summer lose } \\
\text { Woolman's Ifarvest. } \\
\text { Lippincot. }\end{array}$ & $\mathrm{s}$. & -- & & * & * * & \\
\hline 148 & Summer IIagloe & L. S. & к. & & & & .. \\
\hline $\begin{array}{l}149 \\
150\end{array}$ & $\begin{array}{l}\text { Summer Sweet Paradise } \\
\text { Strawn's Seedling ....... }\end{array}$ & S. & K. & -. & & & * \\
\hline $\begin{array}{l}151 \\
152\end{array}$ & $\begin{array}{l}\text { Swain } \ldots+1 \\
\text { Swaar }\end{array}$ & A. & ... & $\ldots$ & & & $\because$ \\
\hline & & $\mathrm{s}$ & . & & & & \\
\hline 154 & $\begin{array}{c}\text { Tinmouth } \\
\text { Teignmouth. }\end{array}$ & W. & & & & & \\
\hline $\begin{array}{l}15.5 \\
156\end{array}$ & $\begin{array}{l}\text { Tolman's Sweeting. } \\
\text { Towne }\end{array}$ & $\begin{array}{l}\text { W. } \\
\text { A. }\end{array}$ & K. M. & - * & * & & $* *$ \\
\hline
\end{tabular}




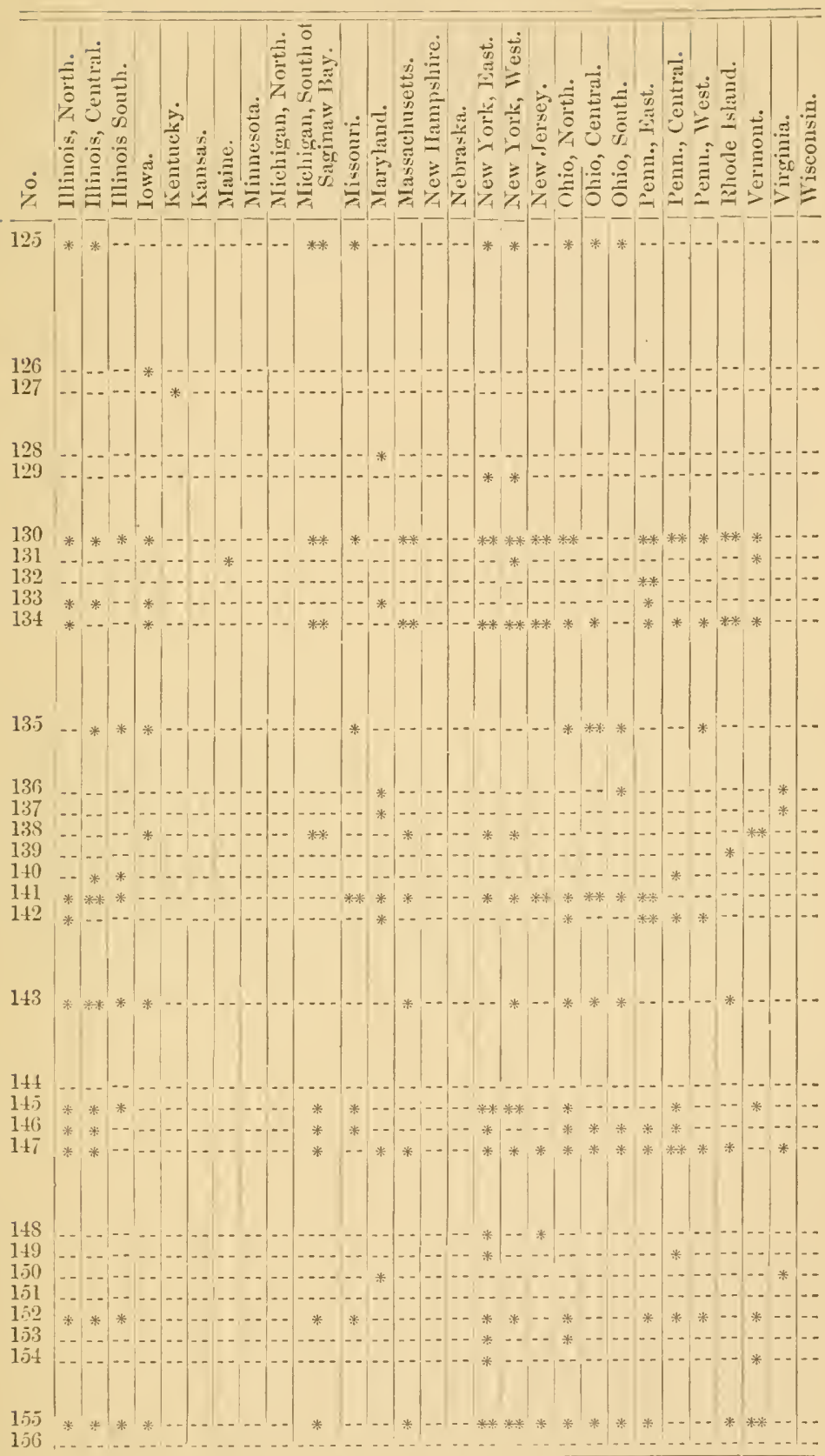




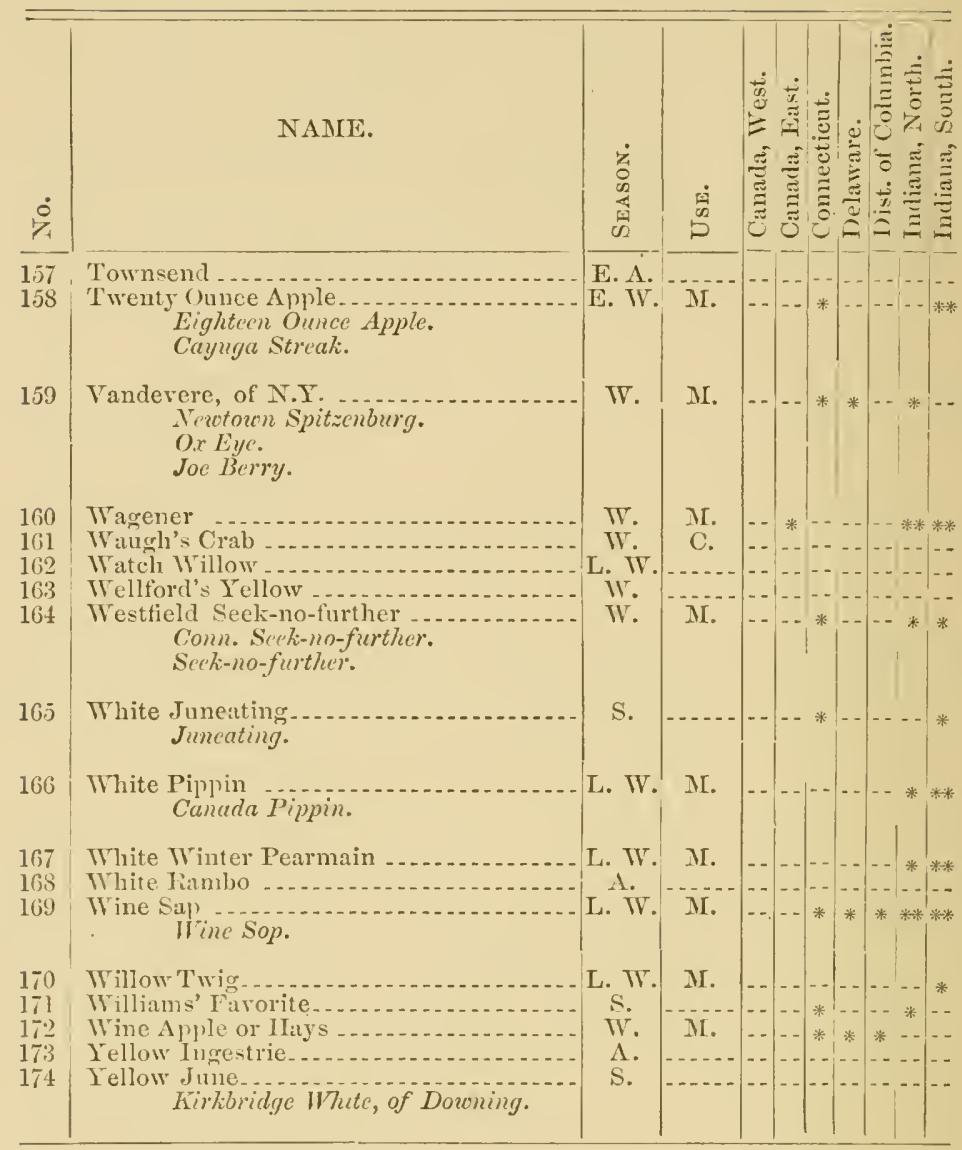




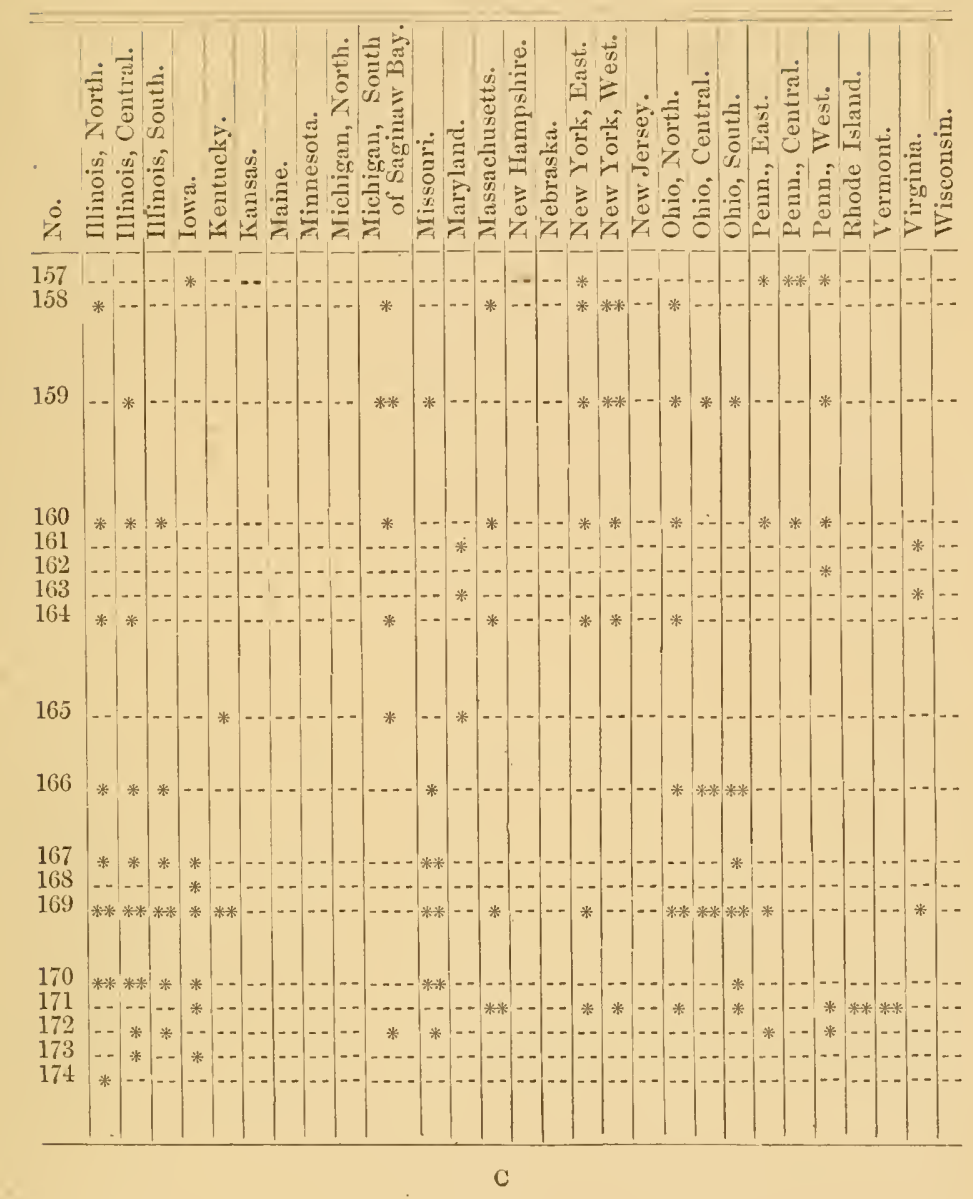





\section{II.-PEARS.}

The Columns indicate-1st. The Season; 2t. Use; 3d. Stock; and the remainder the Districts in which the varieties are recommended.

The abbreviations are-

SEASox.-S. summer; A. antumn; W. winter; E. early; I. late.

USE - K. denotes those recommended only for kitchen purposes; M. those most profitable for market.

STock.-Q. those which are known to succeed well on the Quince stock. 


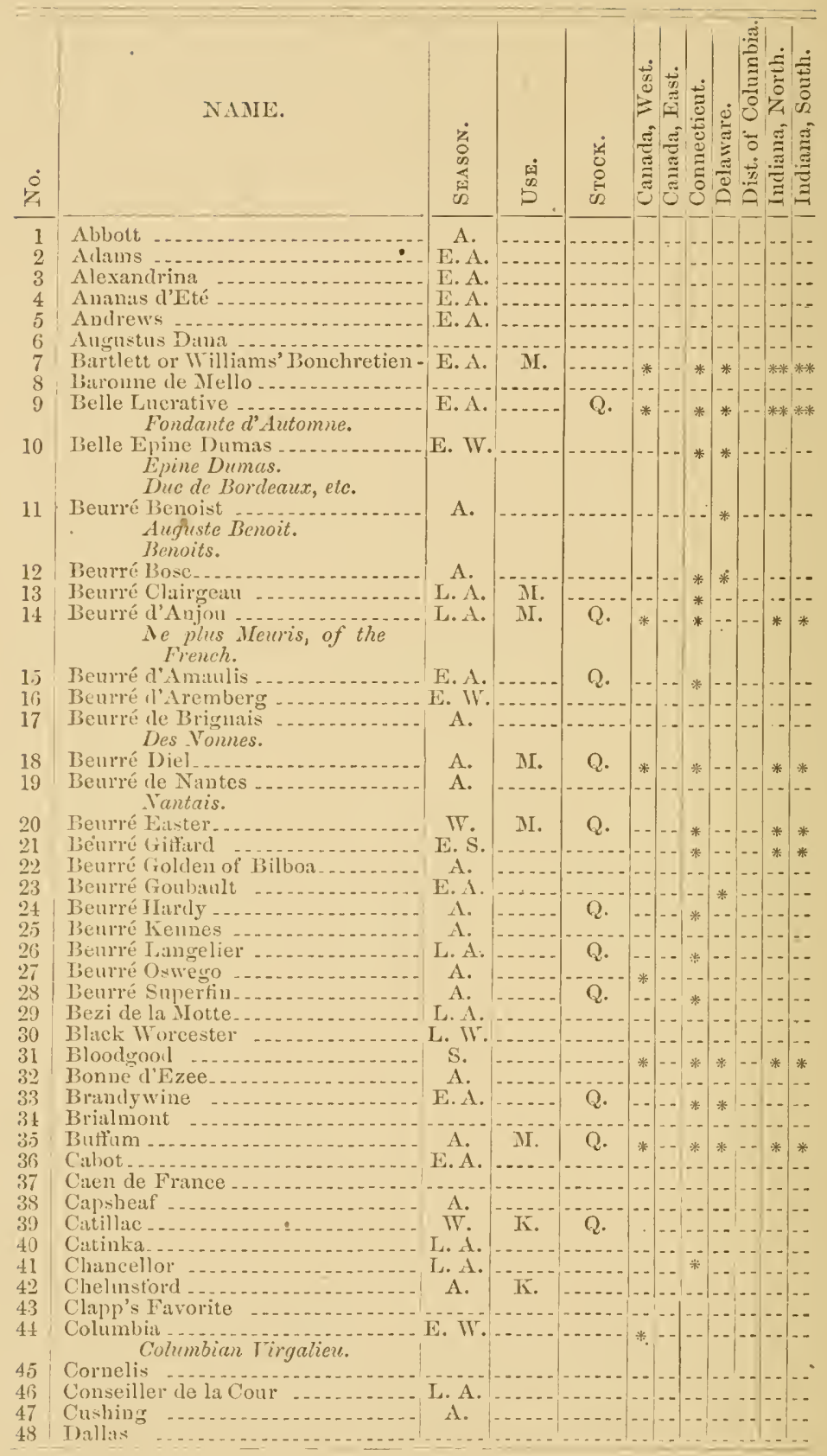




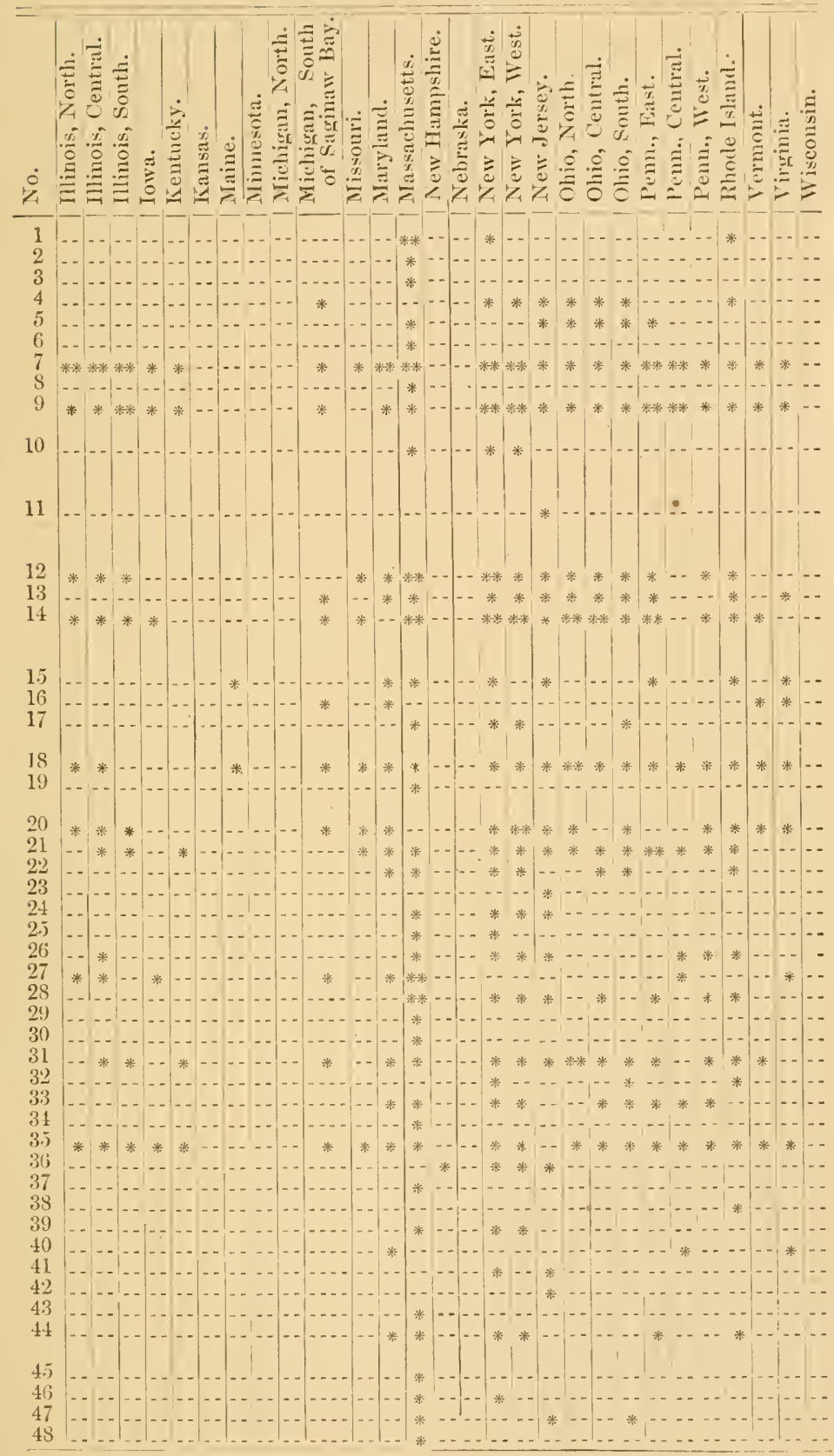




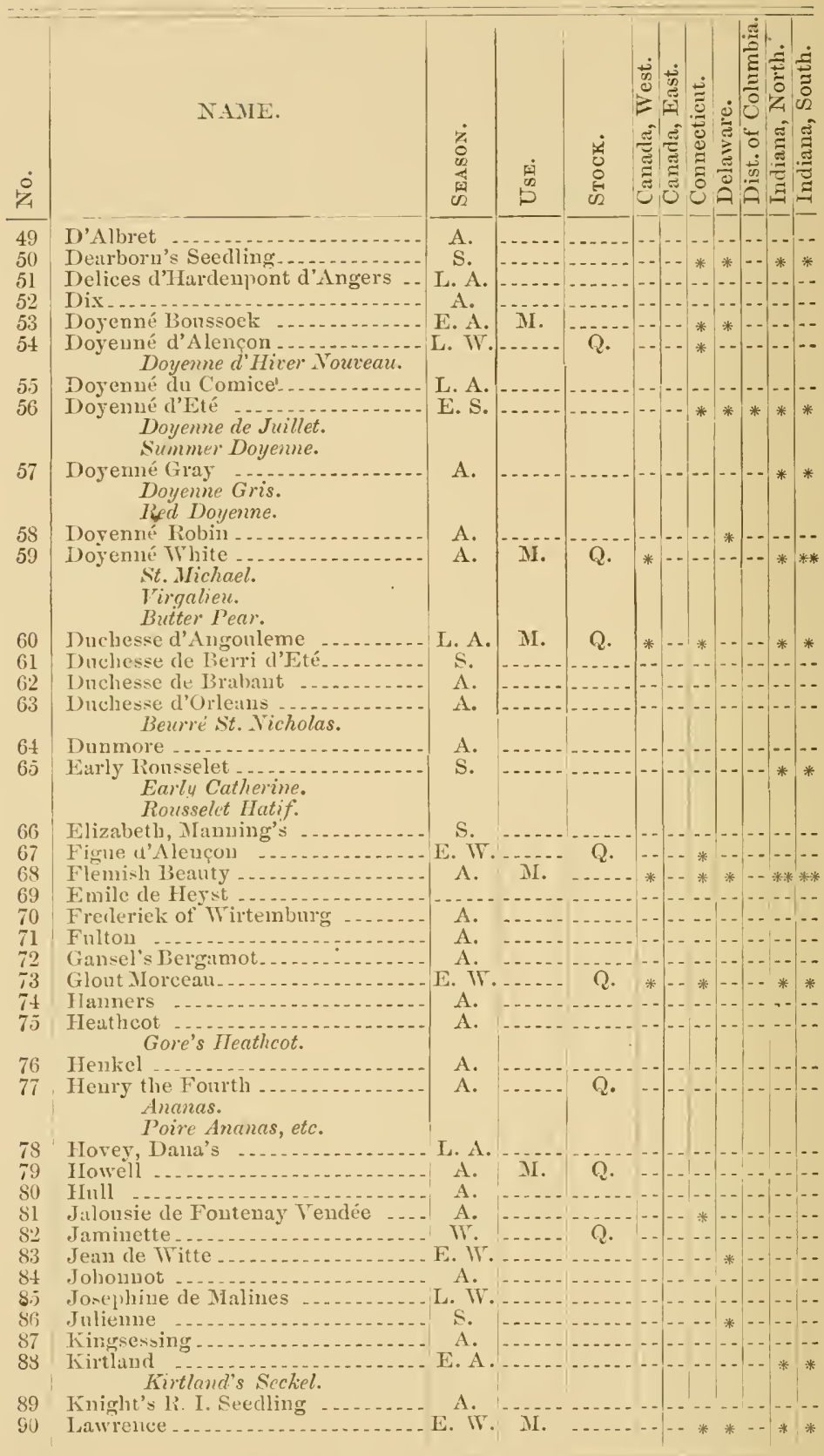




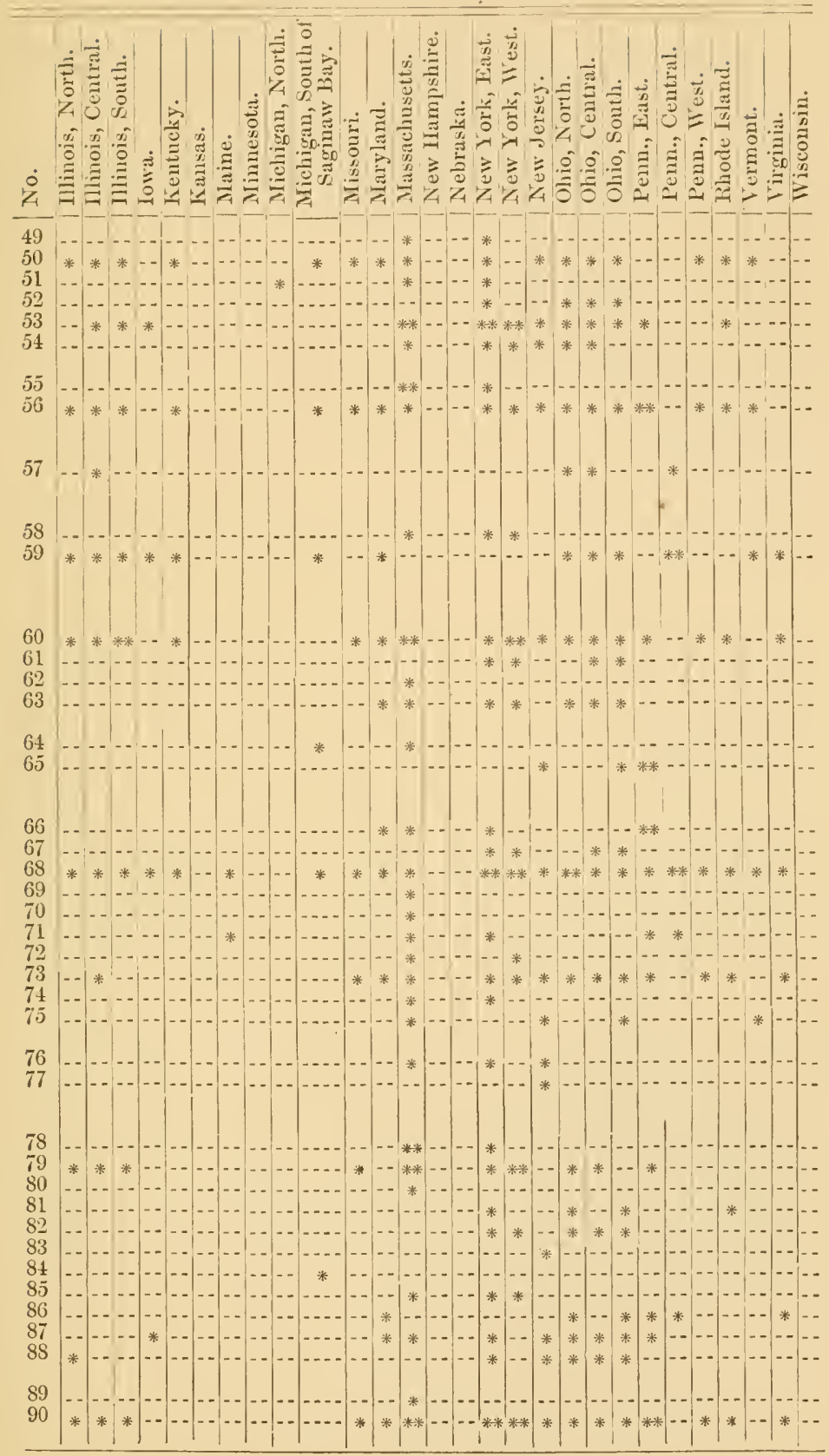




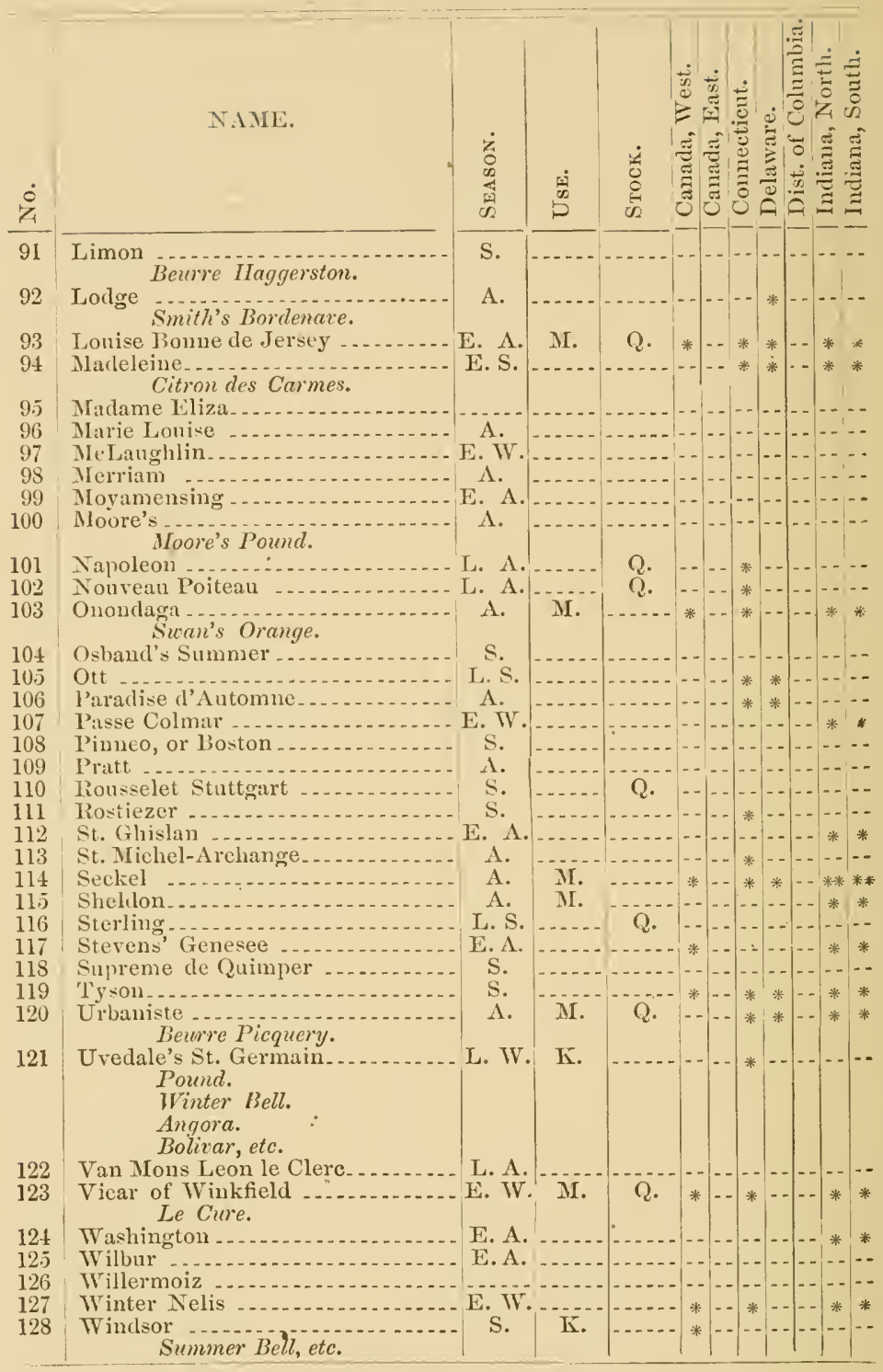




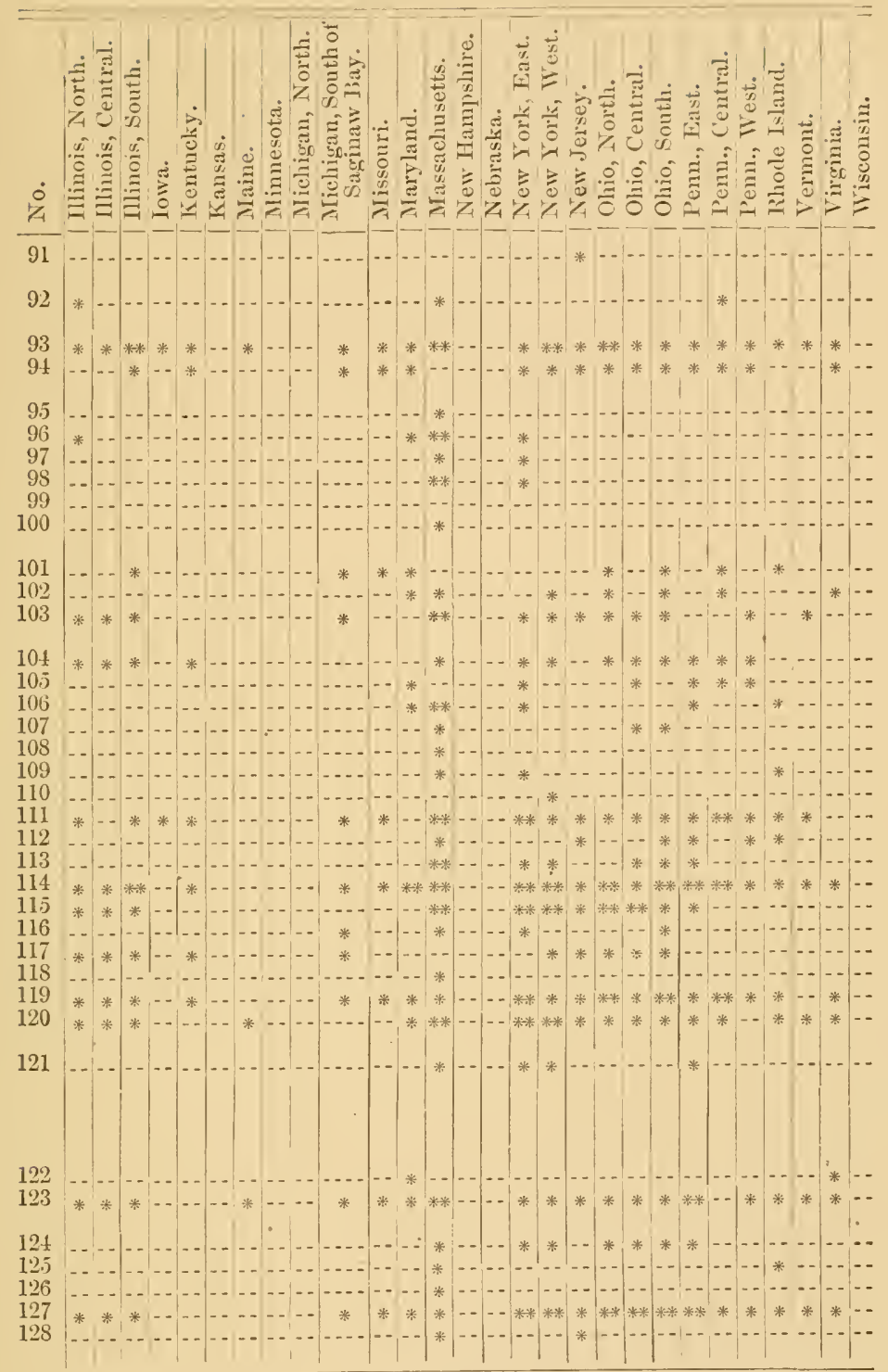





\section{III. - CHERRIES.}

The columns explain - 1st. The season of maturity; $2 \mathrm{~d}$. The class to which the variety belongs; and the remainder the Districts in which the varieties are recommended.

\section{The abbreviations are -}

Seasox. - E. early, as Early Purple Guigne, Belle d'Orleans, Kentish, etc., which usually ripen at Rochester, lat. $43 \mathrm{deg}$., from the 10 th to 20 th of June, according to the season. M. medinm season, those ripening between 20 th of June and 20 th of July; and L. late, those ripening after that time.

CLAss.-H. hearts, or tender-fleshed, sweet cherries, such as Black-Heart or Gov. Wood. B. bigarreau or firm-fleshed, sweet cherries, like Graffion, Napoleon, etc. D. dukes, having more or less acidity, as May Duke. M. MInrellos, having generally acid fruit, used chiefly for kitchen or confectionery purposes. 


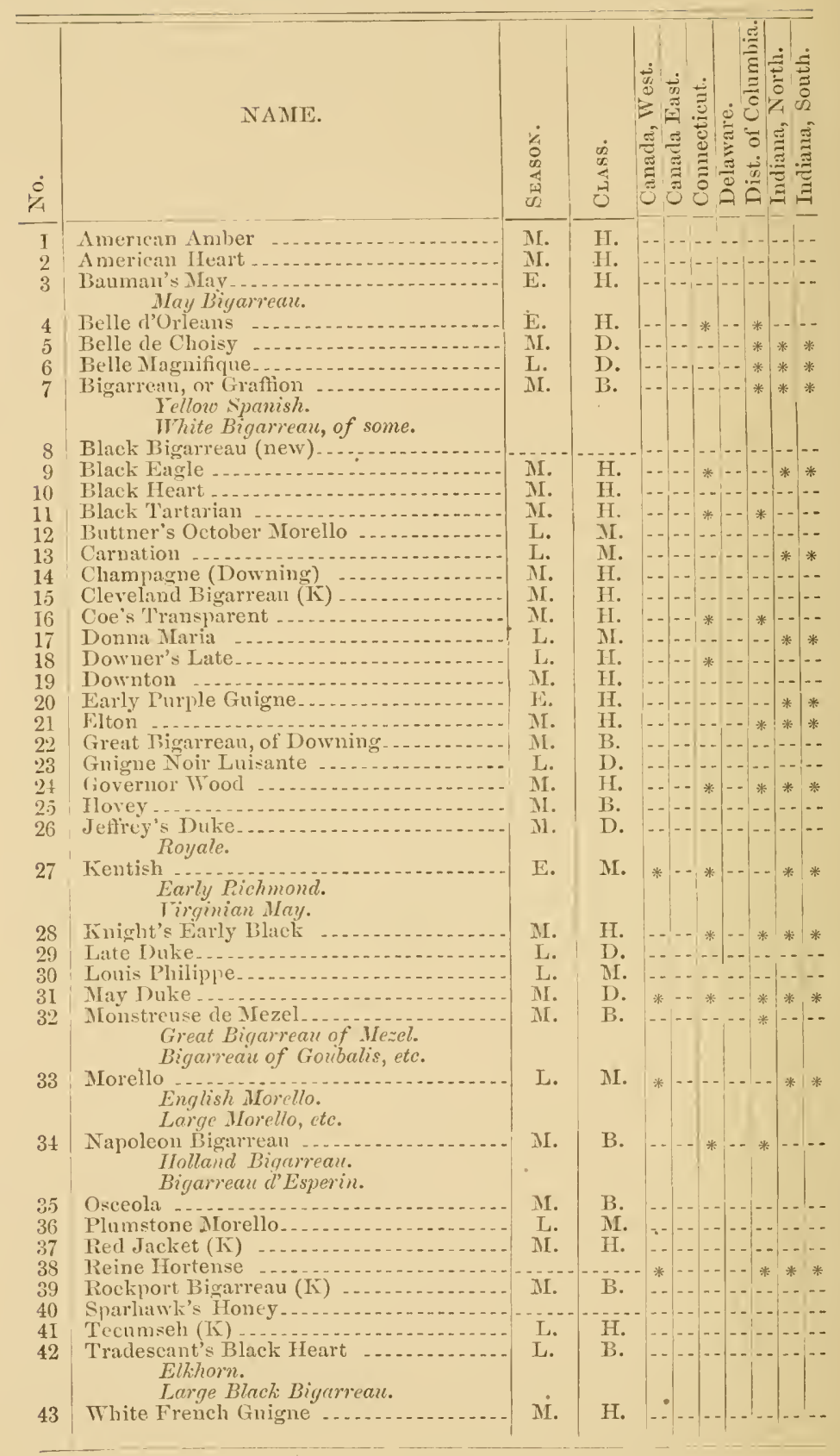




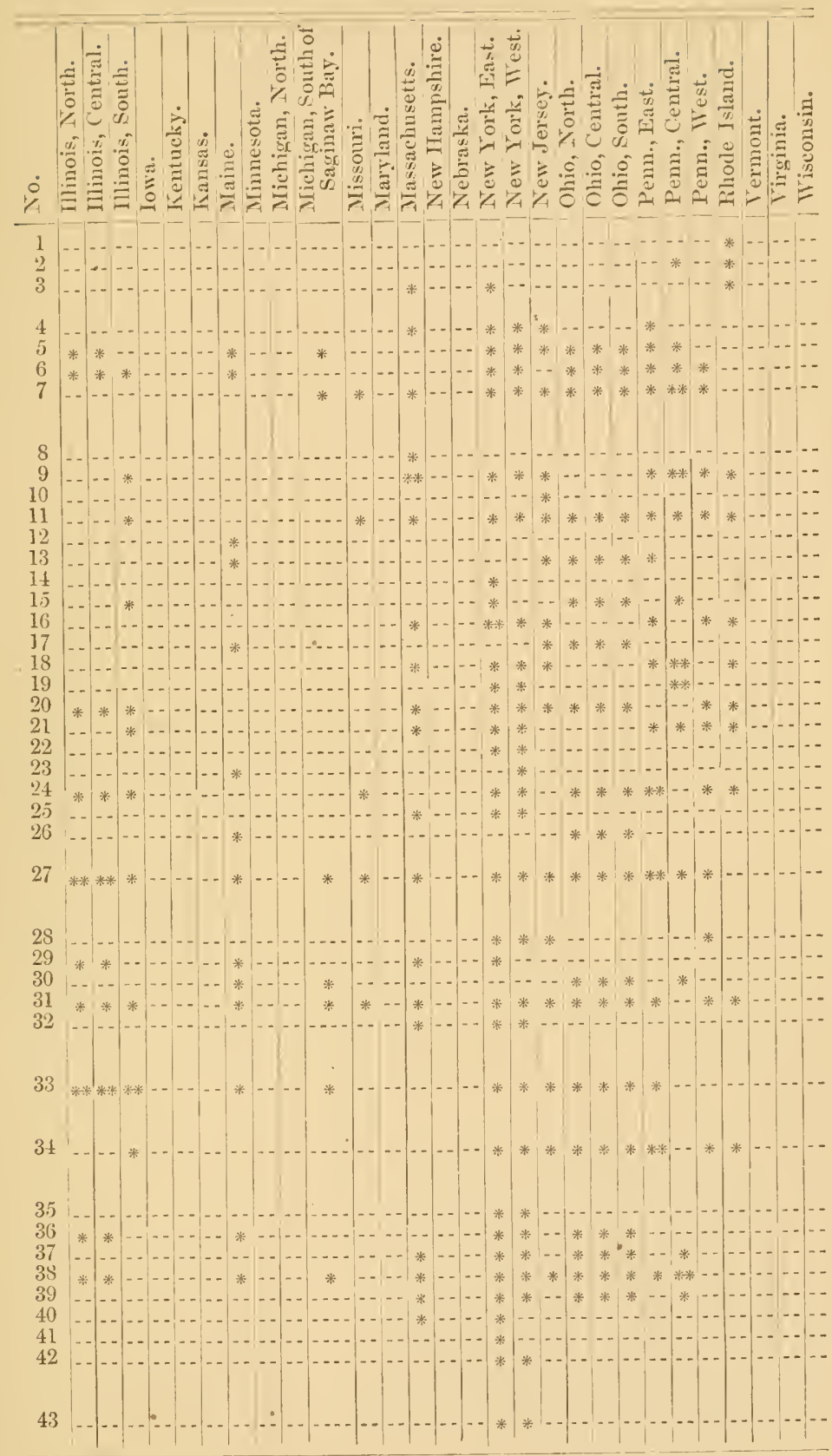





\section{IV. - PEACHES.}

The columns explain:

1st. The class, as Freestone or Clingstone, to which the variety belongs.

$2 d$. The color of the flesh.

3d. The season of maturity; as Early, Medium, or Late. Those designated as Early, ripen in lat. 43 deg., previous to or about Sept. 1st. Medium, those ripening from 1st to 15 th of Sept.; and Late, those after that period. A few of the very early and very late are so designated. The remaining columns denote the Districts in which the varieties are recommended.

\section{Abbreviations :}

Class. - F. freestone; C. clingstone.

Flesh. - IV. white, or pale-colored flesh; $Y$. yellow, or yellowish flesh.

SEAson.-E. early; V. E. very early; M. medium; L. late; V. L. very late. 


\begin{tabular}{|c|c|c|c|c|c|c|c|c|c|}
\hline$\dot{8}$ & NAME. & 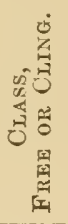 & 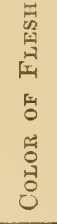 & 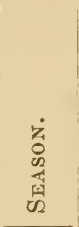 & 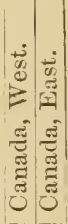 & & & & 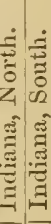 \\
\hline 1 & $\begin{array}{l}\text { Barnard } \\
\text { Early Bamard. } \\
\text { Yellow Bamard. }\end{array}$ & F. & Y. & M. & & & - & & * \\
\hline$\frac{2}{3}$ & $\begin{array}{l}\text { Barrington } \\
\text { Bellegarde }\end{array}$ & $\mathrm{F}$ & IV. & M. & & & & & * \\
\hline $\begin{array}{l}3 \\
4\end{array}$ & Bergen's Yello & $\mathrm{F}$. & Y. & MI. & & & & & \\
\hline 5 & Cambridge Be & $\mathrm{F}$. & IV. & II. & & & - & & \\
\hline 6 & $\begin{array}{l}\text { Carpenter's WV } \\
\text { Cole's Early }\end{array}$ & $\mathrm{F}$. & W. & L. & & & $-x_{2}$ & & \\
\hline $\begin{array}{l}7 \\
8\end{array}$ & Columbia -. & $\begin{array}{l}\text { F. } \\
\text { F. }\end{array}$ & Y. & M. & & & & & \\
\hline & Cool & F. & W. & E. & & & & & * \\
\hline 10 & ord's Ear & $\mathrm{F}$. & $Y$. & E. & 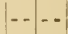 & & * & & * *** \\
\hline & Crawford's Late Mel & F. & $Y$ Y. & L. & & & * & & \\
\hline 12 & Early Newington $\mathrm{Fr}$ & F. & WV. & L. & & & - & & $-{ }^{*}$ \\
\hline 14 & Early Slocum & $\mathrm{F}$. & W. & E. & & & & & \\
\hline 15 & Early Tillotson -.. & F. & W. & V. E. & & & & & 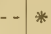 \\
\hline 16 & $\begin{array}{l}\text { Early York } \\
\text { Serrate Early York. } \\
\text { Early Purple. }\end{array}$ & F. & W. & V. E. & & & * & & * * \\
\hline & Fay's Early Au & F. & WV. & V. E. & & & & & *-- \\
\hline 18 & G & F. & & E. & & & * & & \\
\hline & G & C. & W. & I. & & & & & \\
\hline 20 & $\begin{array}{l}\text { Grosse Mignonne - } \\
\text { Uaines' Early Red }\end{array}$ & F. & W. & E. & & & - & * & \\
\hline $\begin{array}{l}21 \\
22\end{array}$ & Hales' Early (from Ohio) & F. & w. & V. E. & & & -1 & & \\
\hline 23 & IIeath Cling & C. & $\mathrm{W}$. & L. & 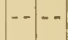 & & $*$ & & \\
\hline & $\begin{array}{l}\text { Mills Madeira Freestone. } \\
\text { Mare }\end{array}$ & & & & & & & & \\
\hline & Hyslop & C. & W. & 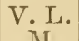 & & & & & \\
\hline 27 & Kemrick's He & F. & W. & V. & & & & & \\
\hline & Heath & & & & & & & & \\
\hline 29 & & F. & WT. & V.L. & & & & * & \\
\hline 30 & Lar & C. & $w$. & I. & & & *. & **. & * \\
\hline $3 l$ & Lat & F. & W. & II. & .. & & * & & -- \\
\hline $\begin{array}{l}32 \\
33\end{array}$ & Lem & C. & Y. & L. & & & & & * \\
\hline 34 & & F. & u & MI. & & & & & * \\
\hline 35 & M & $\mathrm{F}$ & & & .. & & & & \\
\hline 36 & Thite. & F. & W. & II. & $\ldots$ & & * & * & * \\
\hline $\begin{array}{l}38 \\
38\end{array}$ & O & $\mathrm{F}$. & w. & L. & $\because-$ & & * & & * \\
\hline 39 & Cling & C. & W. & L. & & & $*$ & & \\
\hline 40 & $\begin{array}{l}\mathrm{Pr} \\
\mathrm{Re}\end{array}$ & . & & & & & & & $\ldots$ \\
\hline 42 & I & C. & W. & V. L. & & & * & & *. * \\
\hline 43 & & $\mathrm{~F}$ & W. & E. & & & & & * \\
\hline 44 & & & & & & & & & \\
\hline 45 & Freestone & F. & Y. & $\mathrm{L}$. & $\cdots$ & & * & & * \\
\hline 47 & & F & II & I & & & & & \\
\hline 48 & & $\mathrm{~F}$ & Y. & MI. & & & & & \\
\hline 49 & & F. & $Y$. & II. & & & & & 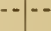 \\
\hline 50 & Tippecanoe Cling & C. & & & & & & & \\
\hline
\end{tabular}




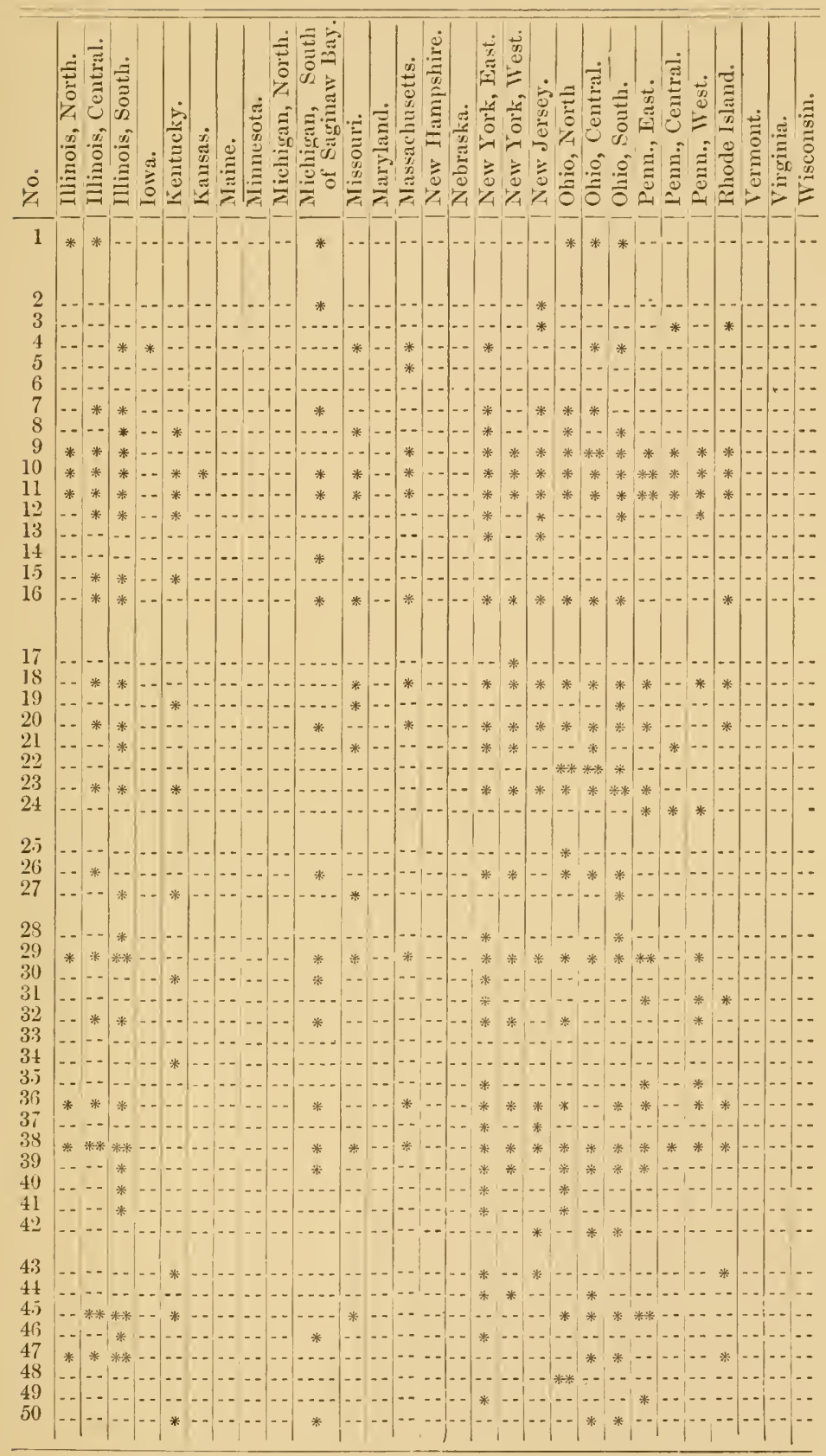




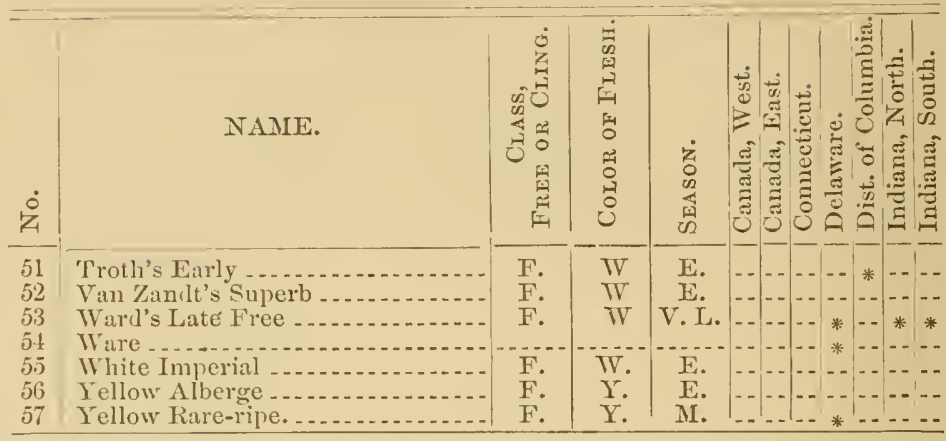

\section{V.- NECTARINES.}

Explanation of columns and abbreviations same as Peaches.

\begin{tabular}{|c|c|c|c|c|c|c|c|}
\hline 7 & NAME. & 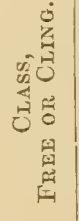 & 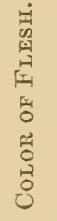 & 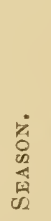 & 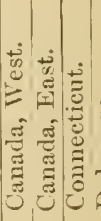 & 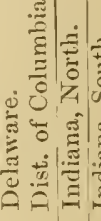 & 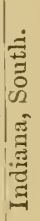 \\
\hline $\begin{array}{l}1 \\
2 \\
3\end{array}$ & 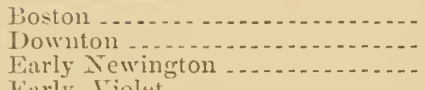 & $\begin{array}{l}\mathrm{F} . \\
\mathrm{F} \\
\mathrm{C} .\end{array}$ & $\begin{array}{l}\text { Y. } \\
\text { IV. } \\
\text { IV. }\end{array}$ & $\begin{array}{l}\text { M. } \\
\text { M. } \\
\text { M. }\end{array}$ & &.- & 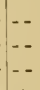 \\
\hline $\begin{array}{l}3 \\
6\end{array}$ & $\begin{array}{l}\text { Larly Vinlet } \\
\text { Violet Ilative. } \\
\text { Elruge } \\
\text { Stanwick }\end{array}$ & $\begin{array}{l}\text { F. } \\
\text { F. }\end{array}$ & $\begin{array}{l}\text { Y. } \\
\text { W. } \\
\text { W. }\end{array}$ & $\begin{array}{l}\text { E. } \\
\text { MI. } \\
\text { L. }\end{array}$ & & * & $\begin{array}{l}* \\
* \\
*\end{array}$ \\
\hline
\end{tabular}




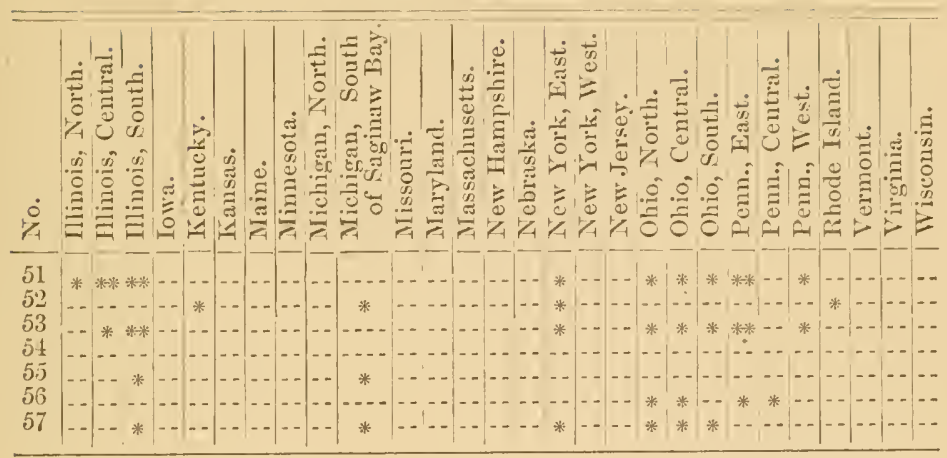

\section{V.-NECTARINES.}

Explanations of columns and abbreviations same as Peaches.

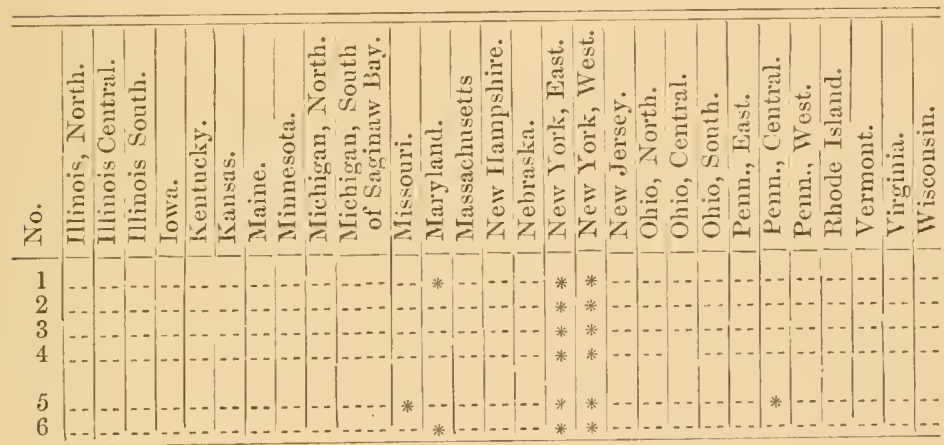




\section{VI. - A PRICOTS.}

Explanation of columns and abbreviations same as Peaches and Nectarines.

\begin{tabular}{|c|c|c|c|c|c|c|c|}
\hline$\dot{0}$ & NAME. & 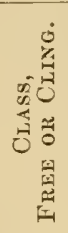 & 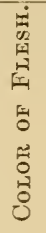 & 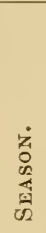 & 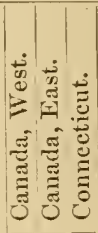 & 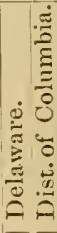 & 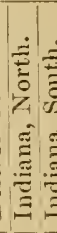 \\
\hline 1 & $\begin{array}{l}\text { Breda } \\
\text { Early } \text { tolden }\end{array}$ & F. & $\mathrm{O}$. & M. & -- & & * \\
\hline 3 & Hunskirke -. & & & & & & \\
\hline 4 & Large Early - . & F. & O. & E. & $\ldots$ & & \\
\hline $\begin{array}{l}5 \\
6\end{array}$ & $\begin{array}{l}\text { Large Red ... } \\
\text { Latayette .... }\end{array}$ & F. & O. & M. & & & \\
\hline 7 & Moorpark .......... & $\mathrm{F}$. & 0. & M. & - & * & * \\
\hline 8 & Orange Royal Orange. & c. & O. & E. & & & \\
\hline 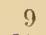 & Peach .............. & F. & Y. & M. & & & \\
\hline 10 & 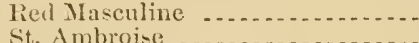 & F. & $Y$. & E. & 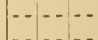 & * & \\
\hline 12 & Turkey & $\mathrm{F}$. & $\mathrm{Y}$. & $\mathrm{L}$. & & & \\
\hline
\end{tabular}




\section{VI. - A PRICOTS.}

Explanation of columns and abbreviations same as Peaches and Nectarines.

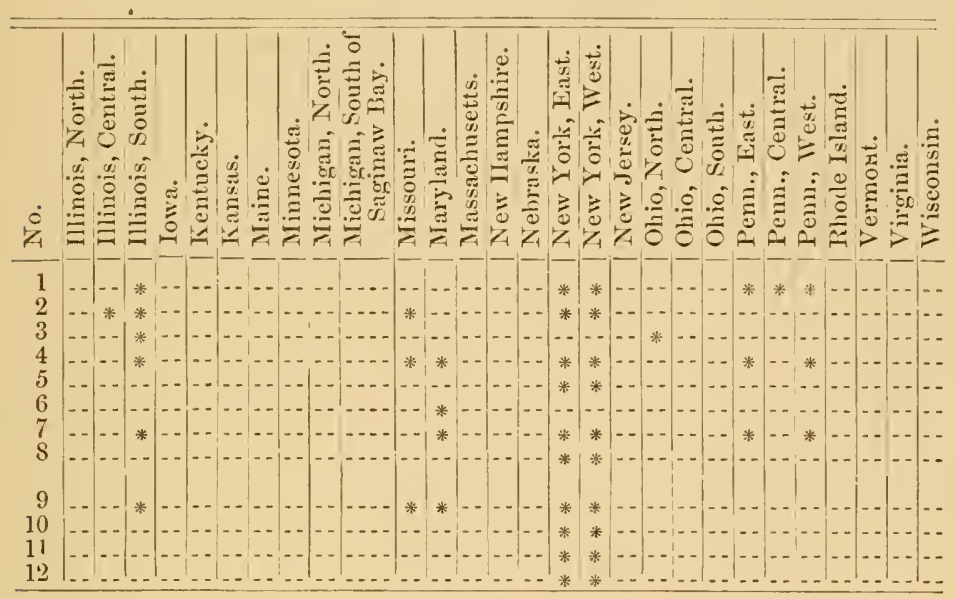





\section{VII.—PLU IIS.}

The columns indicate-1st. Color of Fruit; 2nd. Whether Freestone or Cling; 3d. Uses to which best adapted; 4th. Season of maturity; and the remainder the Districts in which the varieties are recommended.

The abbreviations are as follows:-

CoLon of Frurt.-D. dark, including all red, purple blue, and other darkcolored varieties; P. pale, including the green, yellow, etc.

SToxe. - F. freestone; C. clingstone.

Uses. - T. table; K. kitchen; D. drying; M. those most profitable for market.

Srason.-E. early; M. medium; L. late; V. E. very early; V. L. very late.

The divisions of scason for Peaches apply also to Plums. 


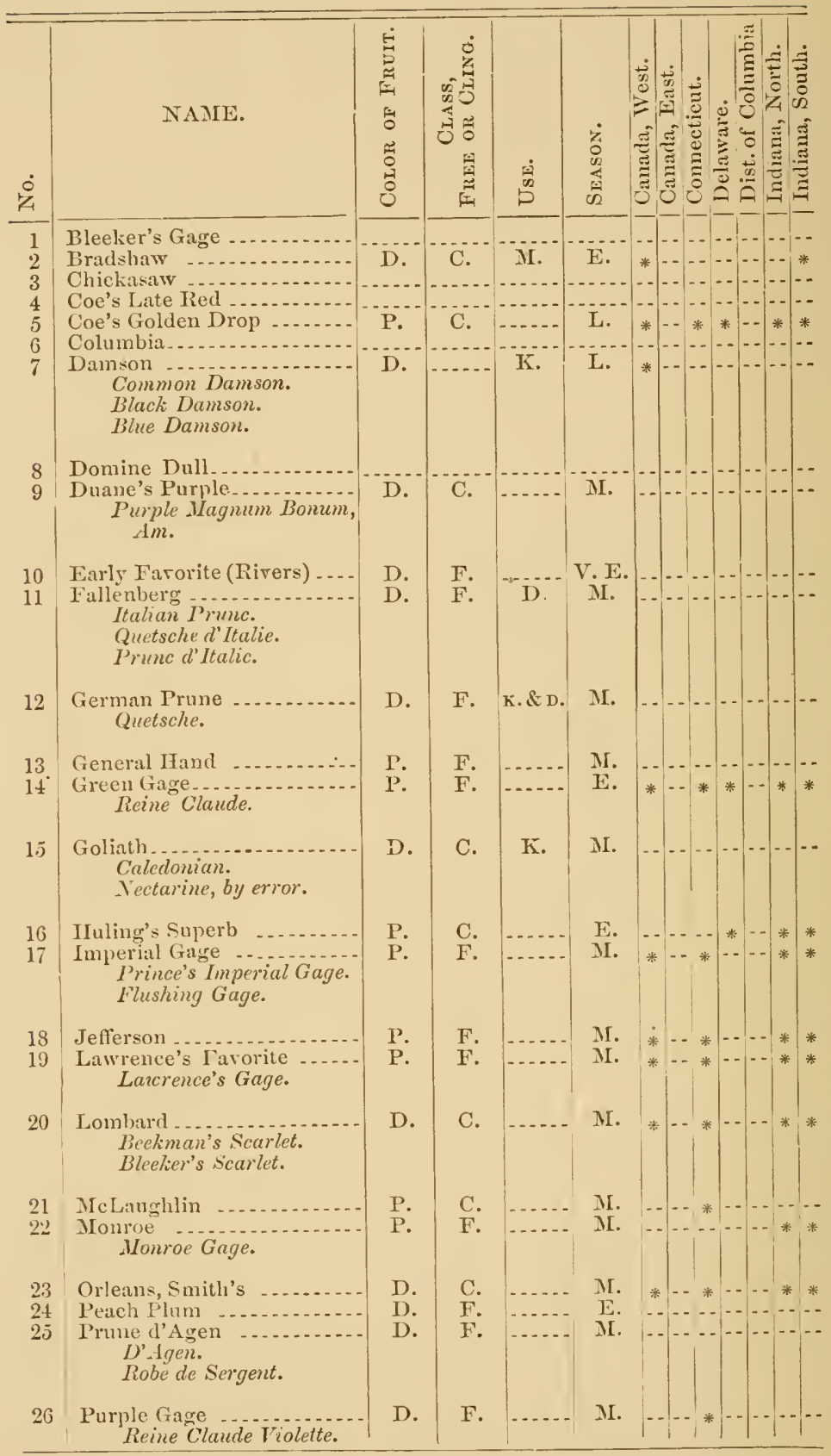




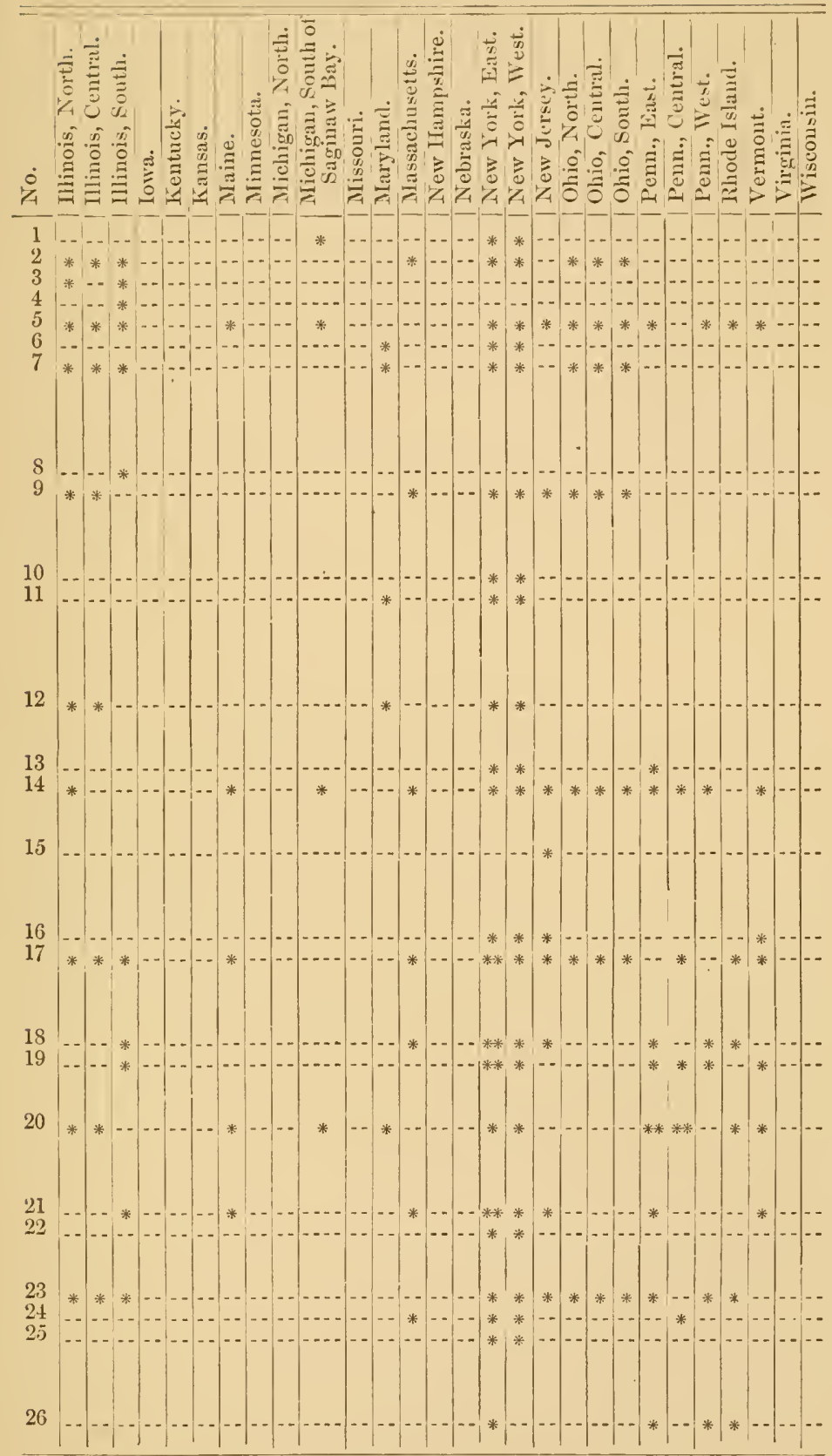




\begin{tabular}{|c|c|c|c|c|c|c|c|c|}
\hline 号 & NAME. & 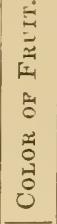 & 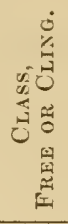 & $\stackrel{9}{\infty}$ & 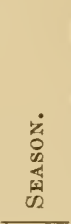 & 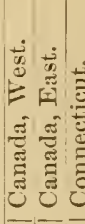 & & 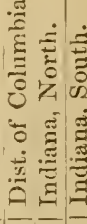 \\
\hline 27 & Purple Farorite. & D. & F. & & E. & & & \\
\hline & Bavay & P. & 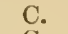 & & L. & .. & & \\
\hline & $\begin{array}{l}\text { Royale Hat } \\
\text { Royale de'I }\end{array}$ & D. & $\begin{array}{l}\text { C. } \\
\text { C. }\end{array}$ & & $\begin{array}{l}\mathrm{E} . \\
\mathrm{E} .\end{array}$ & & & \\
\hline & & $\mathrm{P}$ & C. & & $\mathrm{L}$. & & & \\
\hline & $\begin{array}{l}\text { St. Martin's Quetsche.... } \\
\text { Victoria -.......... }\end{array}$ & P. & F. & & V. L. & & & \\
\hline & $\begin{array}{l}\text { Alderton. } \\
\text { Denyer's Victoria. } \\
\text { Washinuton }\end{array}$ & & F. & & & & & \\
\hline 3 & $\begin{array}{l}\text { Bolmar's. } \\
\text { Bormand }\end{array}$ & 1. & & & E. & & & * \\
\hline 35 & $\begin{array}{l}\text { White Magnum Bonnm ...- } \\
\text { Yellow 1lagnum Bonum. } \\
\text { Yellow Egg. }\end{array}$ & P. & C. & K. & M. & * & & * \\
\hline 36 & Yellow Gage (Prinee's) .... & P. & F. & & E. & * & & 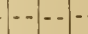 \\
\hline
\end{tabular}

V I I . - Q U I N C E S.

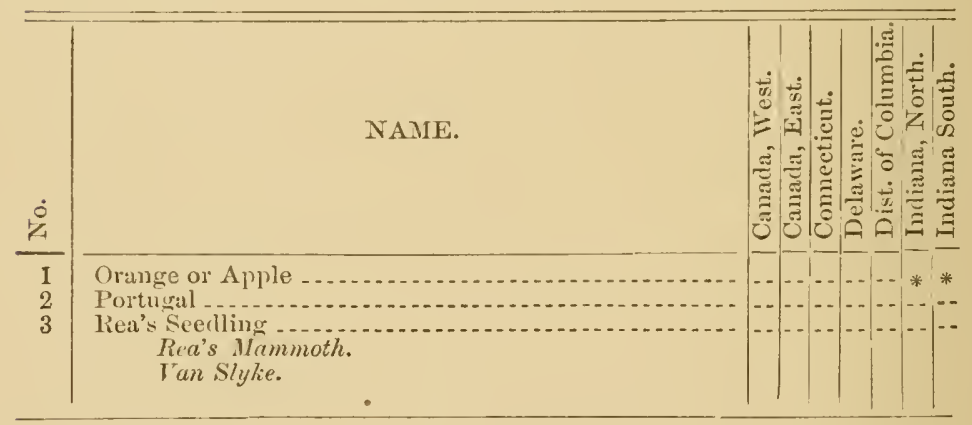




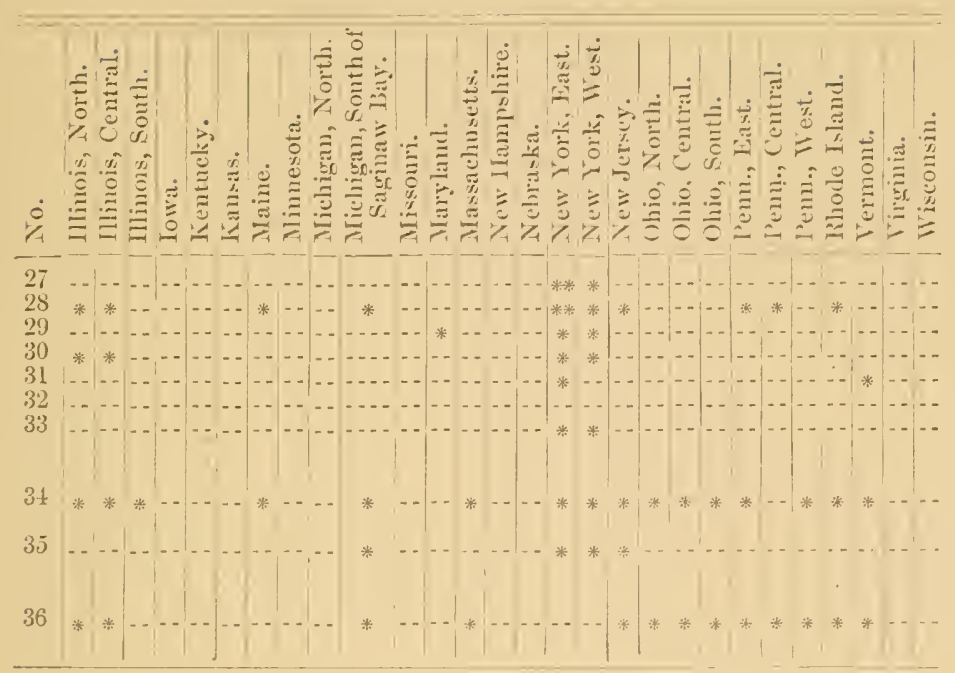

VIII. - Q U I N C E S.

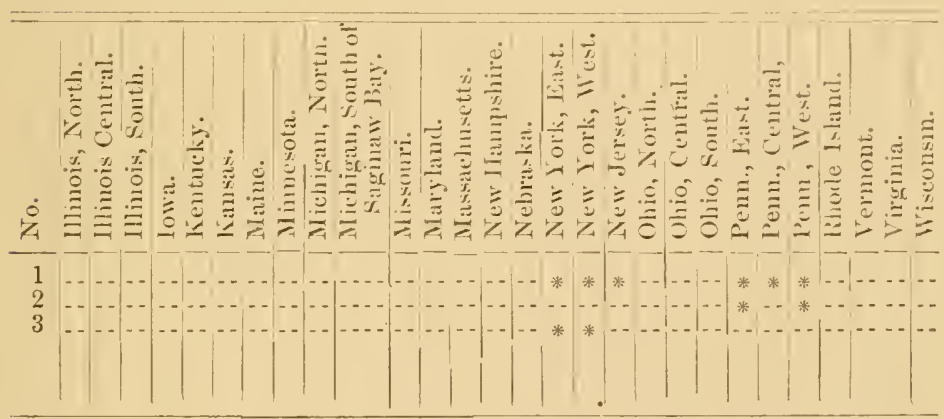




\section{IX.-NATIVE GRAPES.}

The columns explain -1 st. The season; $2 d$. The color; the remainder the Districts where the varieties are recommended.

Abrraviatrons. - Season : E. early; M. medium; L. late. Color: B. black ; R. red; W. white.

\begin{tabular}{|c|c|c|c|c|c|c|c|c|}
\hline$\dot{0}$ & NAME. & $\begin{array}{l}z \\
0 \\
0 \\
4 \\
13 \\
0\end{array}$ & 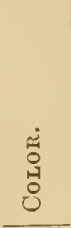 & 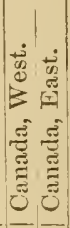 & 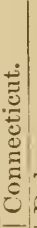 & 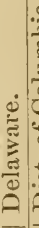 & 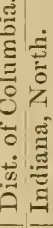 & 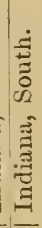 \\
\hline 1 & Adirondack -. & & & & & & & \\
\hline 2 & 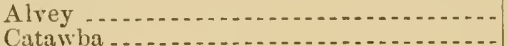 & & & & & - - & & \\
\hline 3 & 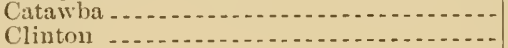 & L. & R. & $\ldots$ & - & $*$ & .. * * & $*$ \\
\hline 4 & 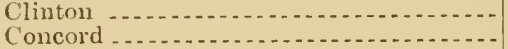 & $\mathrm{E}$ & I & *. & - . & -- & $-\sigma$ & $*$ \\
\hline 5 & & $\mathbf{E}$ & ]. & $* \mid-$ & * & $*$. & $-\cdots *$ & * \\
\hline 6 & (2) & E. & B. &.-- & -- & $-\cdots$ & $-\ldots$ & - \\
\hline 7 & ( & $\mathrm{I}_{2}$ & IV. & $\ldots$ & $\ldots$ & -- & $\ldots$ & - \\
\hline 8 & ( & $\mathrm{I}$ & R. & * & $*$ & $*$ & - - * & $*$ \\
\hline 9 & 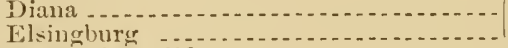 & M. & R. & $--1-$ & * & -- & - * * & $*$ \\
\hline 10 & 1 Prolific. & L. & B. & $--\mid-$ & -- & -- & -- & \\
\hline 11 & l'rolific. & E. & B. & * - & $*$ & -- & -- & - \\
\hline 12 & . & & & (-) & -- & $-\cdot$ & & -- \\
\hline 1 & 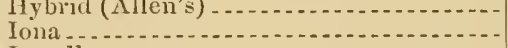 & 1 & . & & - & -- & & - \\
\hline 15 & la & 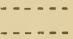 & & & - & -- & & - \\
\hline 16 & - & M. & B. & $\ldots$ & * & * & - - * & * * \\
\hline 17 & -...... & E. & I3. & $\ldots$ & $1-$ & $\ldots$ & & $\ldots$ \\
\hline 18 & . & & IV. & - & $\cdots$ & *. & & \\
\hline 19 & Muscad & $\mathrm{F}$ & li. & .. & 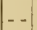 & & & - \\
\hline 20 & " & M. & I3. & -- & . & .. & - - - - & $\ldots$ \\
\hline 21 & Rebeca & E. & WV. & & * & -. & -- & - \\
\hline $\begin{array}{l}22 \\
23\end{array}$ & 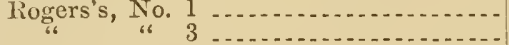 & 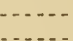 & & & & & & \\
\hline 21 & “ & & & & & & & \\
\hline 2 & y & & & & & & & \\
\hline 2 & " 15 - & & 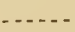 & ... & & - & & \\
\hline$?$ & " $19 \ldots$ & & & & & & & \\
\hline 2 & . & L. & B. & 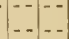 & & & & \\
\hline 30 & Union Village & L. & B. & & & & & \\
\hline
\end{tabular}




\section{IX.-NATIVE GRAPES.}

'The columns explain -1 st. The season; $2 d$. The color; the remainder the Districts where the varieties are recommended.

Ambrevintrons. - Season : E. early; MI. medium; L. late. Color : B. black ; R. red; W. white.

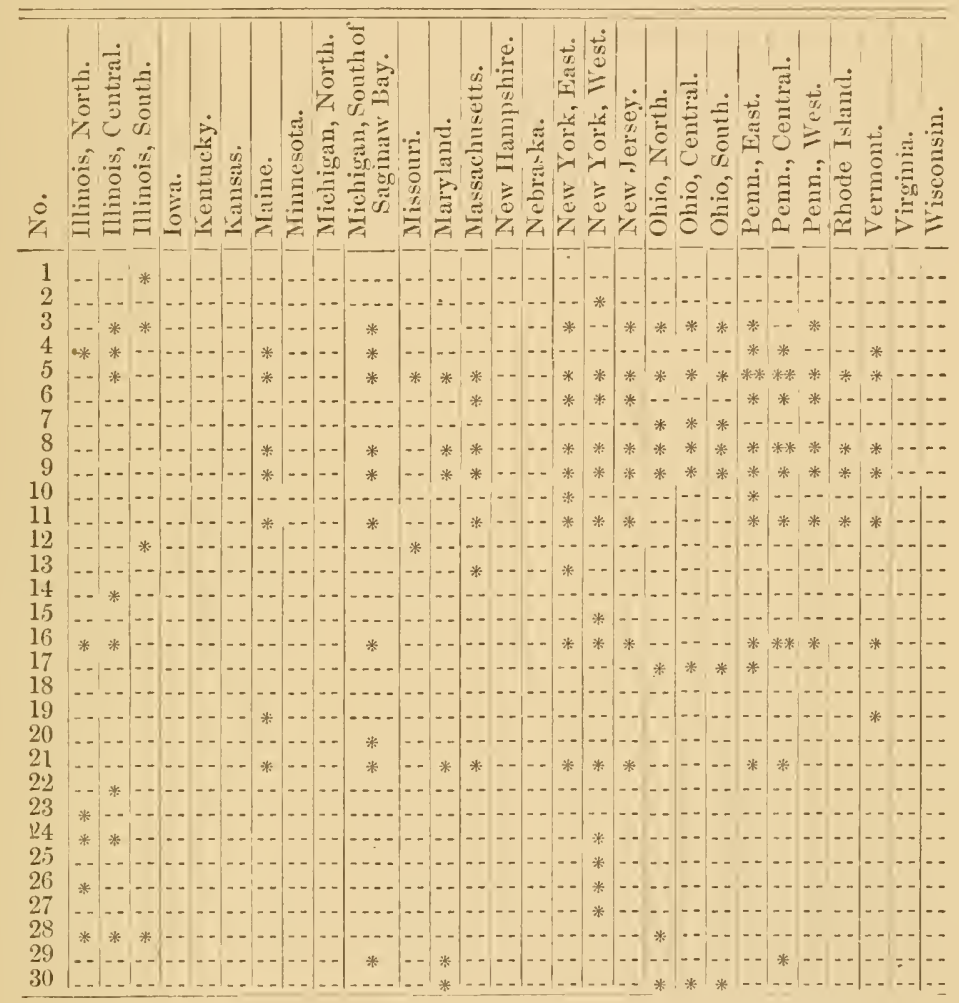




\section{FOREIGN GRAPES.}

As the Foreign Grapes are for cultivation under glass, they are not subject to those variations induced by climate or soil, and, therelore, they may be regarled as equally arlapted to all localities. Very few of the local committees have made any report in reference to these Grapes. The list below contitius such as have been already adopted by the Society, with a few other's vury generally esteemed.

The columns explain - 1st. The color of the fruit; 2l. Flawor; 3il. Suison of maturity. In flavor the only distinction is between thore that are simply sweet, as the Chasselas or Hamburgs, and those having a distunct musky aroma, as the IIuscats.

\begin{tabular}{|c|c|c|c|c|}
\hline$\dot{8}$ & NAME. & CoLOR. & Fla For. & SEASON. \\
\hline 1 & $\begin{array}{c}\text { Barbarossa } \\
\text { Prince Albert. } \\
\text { Brizzola. }\end{array}$ & Black. & Sweet. & Very Late. \\
\hline 2 & Black I Hunaseus . . . . . & Black. & Sweet. & Late. \\
\hline 3 & Black Froutignan. . . . . . . . . . & Jilick. & Muscat. & Lalle. \\
\hline 4 & Black IIamburg ......... & Black. & Sweet. & Mecliuin. \\
\hline$\overline{5}$ & Black Prince...... & Black. & Sweet. & Melium. \\
\hline 6 & Bowood Muscat ......... & White. & Musciat. & Medium. \\
\hline 7 & Bucklaul sweet Water ......... & White. & Sweet. & Medium. \\
\hline 8 & $\begin{array}{c}\text { Calabrian Raisin } \\
\text { Raisin de Calabre. }\end{array}$ & White. & Sweet. & Late. \\
\hline 9 & Cannou tlall MIsscat & WV hite. & Muscat. & Late. \\
\hline 10 & $\begin{array}{c}\text { Chasielas Musqué, or Joslin's St. } \\
\text { Alban's . } \\
\text { Muscat blanc IIative? }\end{array}$ & White. & Muscat. & E:wly. \\
\hline 11 & $\begin{array}{c}\text { Golden IIambures } \\
\text { Ntockwood G. Hamburg. }\end{array}$ & White. & Sweet. & Late. \\
\hline 12 & $\begin{array}{l}\text { Grizzly Frontignan............. } \\
\text { Red Frontignan. } \\
\text { Red Constantia. }\end{array}$ & $\begin{array}{c}\text { Red and Yel- } \\
\text { low. }\end{array}$ & Muscit. & Medium. \\
\hline 13 & Lady Down's Seedling . . . . . . . . . & Black. & Sweet. & Very Late. \\
\hline 14 & Muscat of Alexamdria... & White. & MIuscat. & Liate. \\
\hline 15 & Jinscat IJamburg . . & Black. & Muscat. & Mrectium. \\
\hline 16 & $\begin{array}{l}\text { Red Chasselits } \\
\text { Ruse Chasselas. }\end{array}$ & Red. & Sweet. & Mednum. \\
\hline 17 & Thite Nice... & White. & Sweet. & Late. \\
\hline 18 & West St Peter's & Black & Swee & Very Late \\
\hline 19 & $\begin{array}{c}\text { Wilmot's Black Hamburg.-..... } \\
\text { Dutch IIamburg. }\end{array}$ & Black. & Sweet. & Vledium \\
\hline 20 & $\begin{array}{c}\text { White Sweet Water } \\
\text { Dutch Swoet Water, etc. }\end{array}$ & White. & Sweet. & Early. \\
\hline 21 & $\begin{array}{c}\text { White Frontignan } \\
\text { IVhite Constantia. } \\
\text { Muscat Blanc. }\end{array}$ & White. & Muscat. & Medium. \\
\hline 22 & 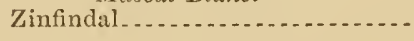 & Black. & Sweet. & Medium. \\
\hline
\end{tabular}





\section{XI. - CURRANTS.}

\begin{tabular}{r|c|c|c|c|}
\hline & & \\
& & &
\end{tabular}

XII. - GOOSEBERRIES.

\begin{tabular}{|c|c|c|c|}
\hline 官 & NAME. & $\begin{array}{l}\text { 官 } \\
\dot{0} \\
8\end{array}$ & 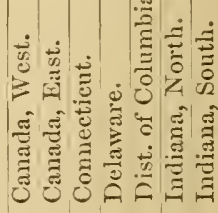 \\
\hline $\begin{array}{r}1 \\
2 \\
3 \\
4 \\
0 \\
6 \\
7 \\
8 \\
9 \\
10 \\
11 \\
12 \\
13\end{array}$ & 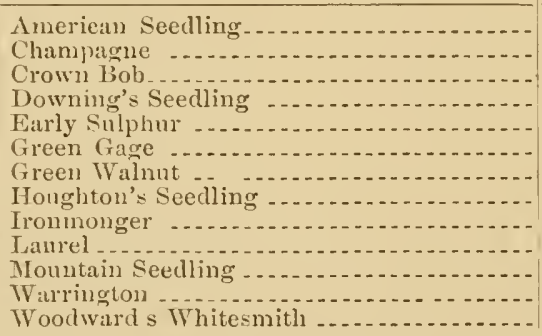 & $\begin{array}{l}\text { Red. } \\
\text { Red. } \\
\text { Red. } \\
\text { Green. } \\
\text { Yellow } \\
\text { Green. } \\
\text { Green. } \\
\text { Red. } \\
\text { Red. } \\
\text { Green } \\
\text { Redl. } \\
\text { liedl. } \\
\text { White. }\end{array}$ & 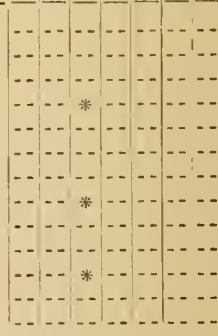 \\
\hline
\end{tabular}

NoTE. - The varieties not reeommented for any particular loeality are those which have been placed on the list for "general cultivation" in the Society's Catalogue for 1860. 
XI. - CURRANTS.

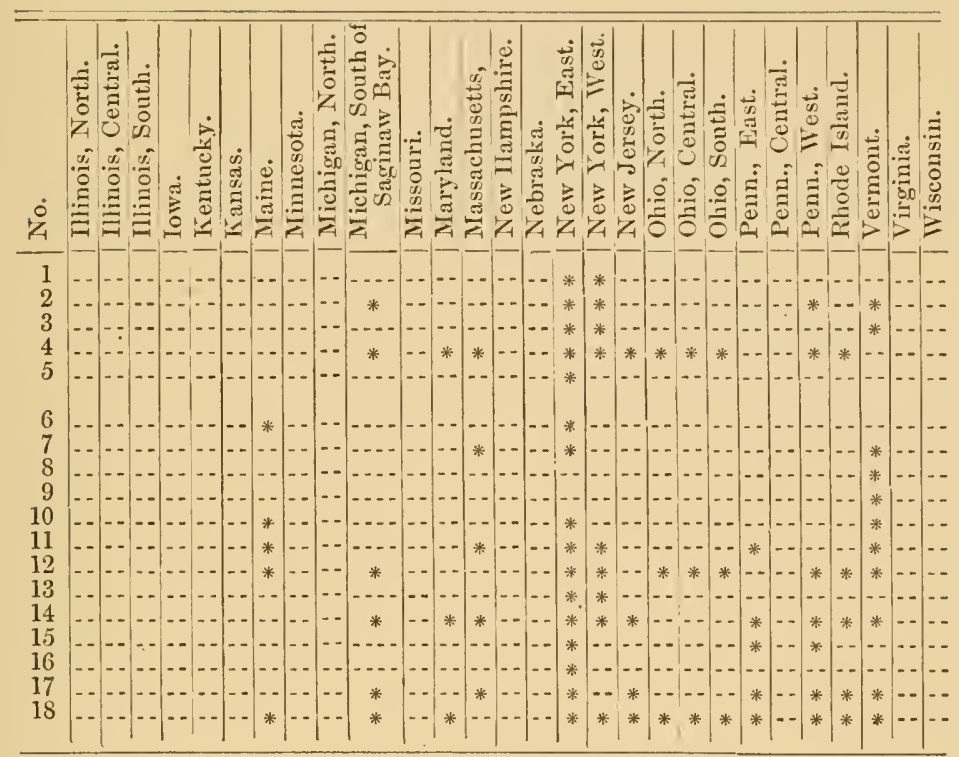

XII. - G O O E B E R R E S.

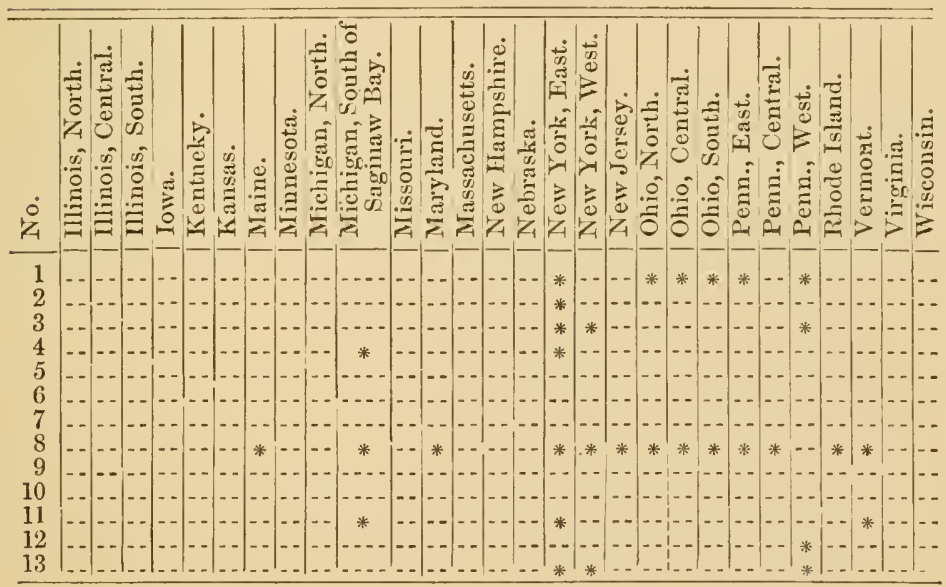


XIII.-RASPBERRIES.

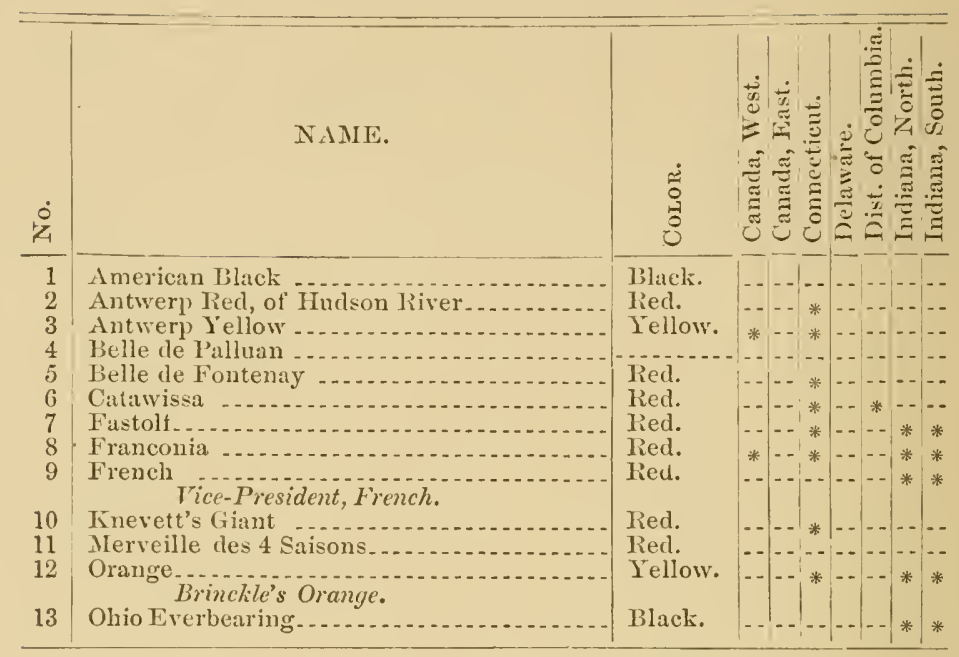

XIV.-BL A CKBERRIES.

\begin{tabular}{|c|c|c|c|c|}
\hline$\dot{0}$ & NAME. & 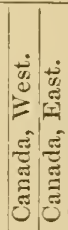 & 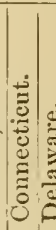 & 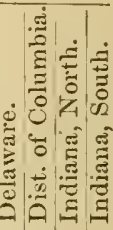 \\
\hline $\begin{array}{l}1 \\
2 \\
3 \\
4\end{array}$ & 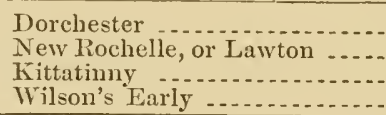 & 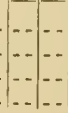 & $\begin{array}{ll}* & * \\
* & * \\
- & * \\
- & \end{array}$ & 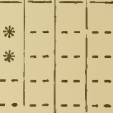 \\
\hline
\end{tabular}


XIII.-R A S PBERIES.

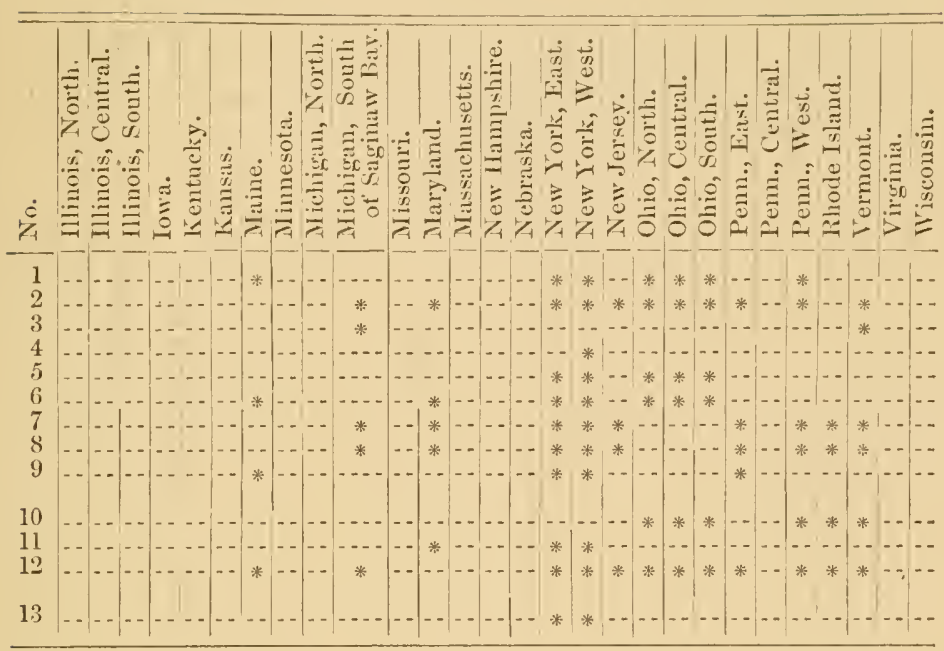

XIV.-BLA CKBERRIES.

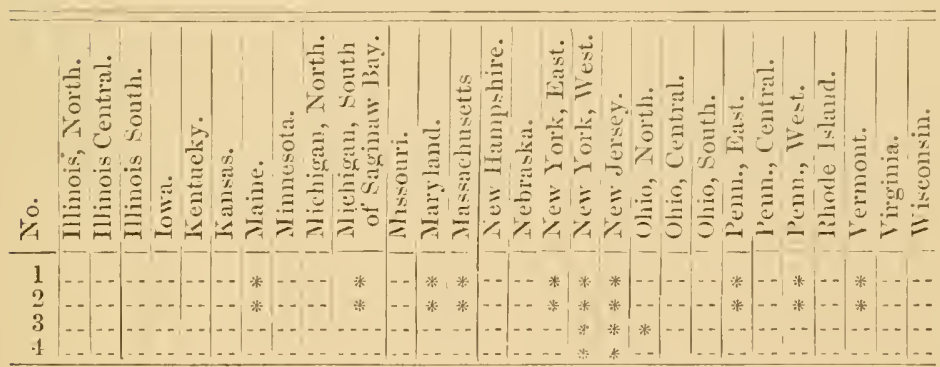




\section{XV. - STRA IV B ERRIES.}

The columns indicate - 1st. The Sex, as Hermaphrodite or Pistillate; $2 d$, The Origin, American or Foreign; and the remainder the districts in which the varieties are recommencled.

The abbreviations are: SEx - P., Pistillate; all others are Hernaphrodite. Origin - A., American; F., Foreign.

\begin{tabular}{|c|c|c|c|c|c|c|c|c|}
\hline$\dot{0}$ & NAME. & 竞 & 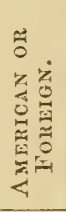 & 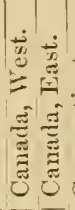 & 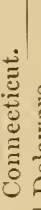 & & 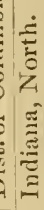 & 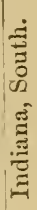 \\
\hline 1 & Alice Maude & & F. & $\ldots$ & - & & * & -. \\
\hline 2 & Brishton Pine. & & A. & $\ldots$ & - & & $-1-$ & $\ldots$ \\
\hline 3 & British Queen..... & & F. & -- & 1 & &.- & .. \\
\hline 4 & Boston Pine ..... & & A. & 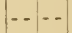 & - & & -. & - . \\
\hline 5 & Burr's New Pine & $\mathrm{I}$ & A. & $*$ & & & * & * \\
\hline 6 & Crimson Cone & $P$ & A. & - & & & $--\cdot$ & - \\
\hline 7 & $\begin{array}{l}\text { Downer's Prolific . . . } \\
\text { Fillmore }\end{array}$ & & A. & $\cdots$ & & & $*$ & -- \\
\hline $\begin{array}{l}8 \\
9\end{array}$ & $\begin{array}{l}\text { Fillmore } \\
\text { Genesee. }\end{array}$ & & $\begin{array}{l}\text { A. } \\
\text { A. }\end{array}$ & $\cdots$ & & & $-\ldots$ & $-\cdots$ \\
\hline 10 & Ilooker ...... & & A. & - & $\ldots$ & & * & * \\
\hline 11 & IHorey's Seedling - & P. & A. & & * & & * $\quad *$ * & * \\
\hline 1 & Iowa $\ldots \ldots \ldots$ & & A. & 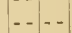 & * & & -- & $-\cdots$ \\
\hline $1:$ & Jenny's Seedling .. & P. & A. & - . & $\cdots$ & &... &.- \\
\hline 1 & Jenny Lind ...... & - & A. & $*-$ & * & $\ldots * *$ & * . . &.- \\
\hline 1 & La Constante ....... & & F. & -- & .. & $\ldots-$ & $\ldots$ &.- \\
\hline 1 & Large Early Scarlet - & & A. & - & -. & &.-- & - \\
\hline 1 & $\begin{array}{l}\text { Longworth's Prolific. } \\
\text { McAroy's Superior. }\end{array}$ & & A. & $\cdots$ & * & & $-*$ & * \\
\hline 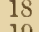 & $\begin{array}{l}\text { McAroy's Superior } \\
\text { Monroe Scarlet }\end{array}$ & P. & A. & $\cdots$ & * & & - * & * \\
\hline 1 & $\begin{array}{l}\text { Monroe Scarlet .... } \\
\text { Moyamensing . ..... }\end{array}$ & $\mathrm{P}$. & A. & - & * & & - & \\
\hline 2 & Sco & & A. & - & * & & ( & \\
\hline & Triomphe de Gand & & $\mathrm{F}$. & * & * & * & * & \\
\hline 2 & Trollope's Victoria . & & F. & - & * & & * . . & \\
\hline 2 & IV alker's Seedling......... & & A. & - - & $*$ & & 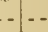 &.- \\
\hline & 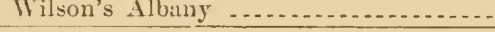 & & A. & & & & & \\
\hline
\end{tabular}




\section{V. - S TR A WBERR E S.}

The columns indicate - 1 st. The Sex, as Hermaphrodite or Pistillate; 2 d. The Origin, American or Foreign; and the remainder the districts in which the varicties are recommended.

The abbreviations are: SEx - P., Pistillate; all others are Hermaphrodite. Origin - A., American; F., Foreign.

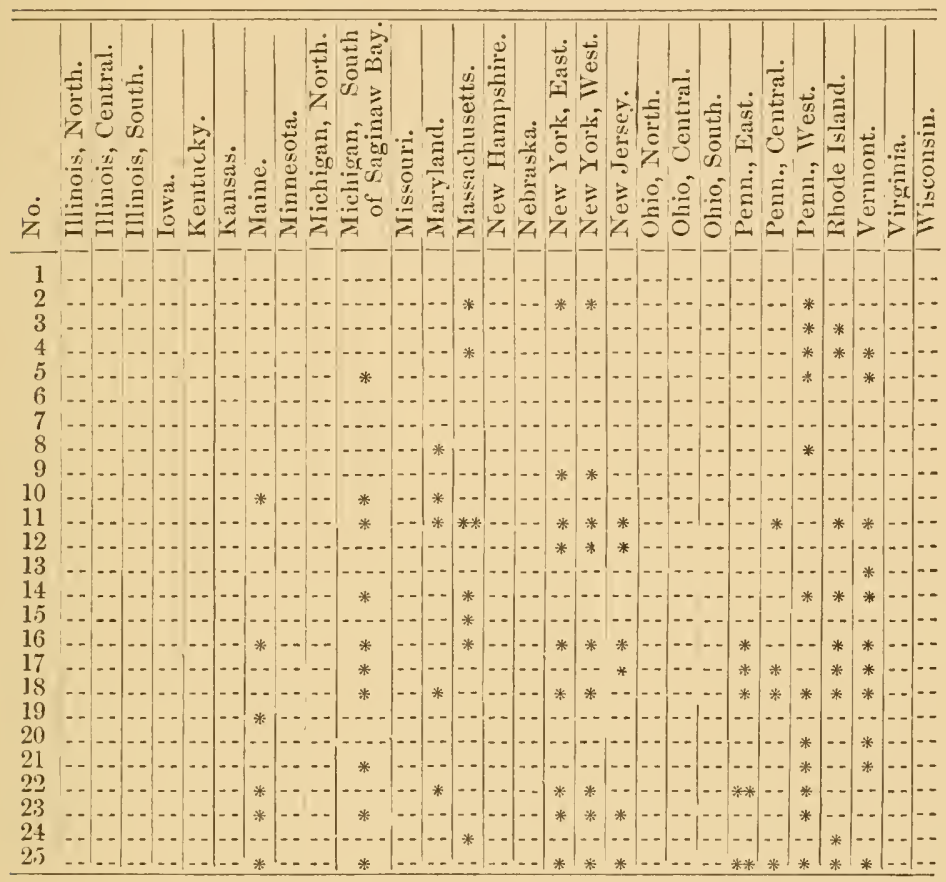






